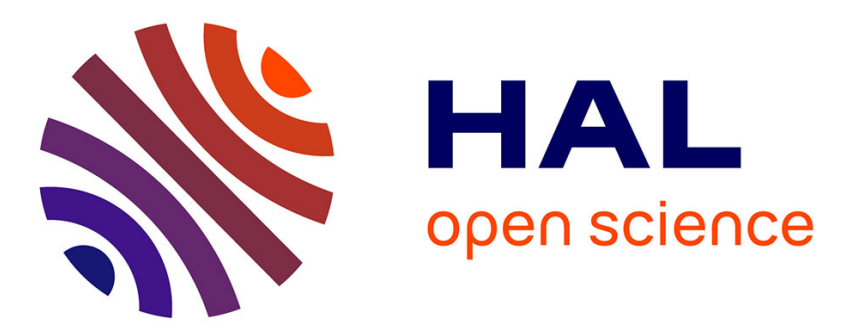

\title{
Optimal control for an organic rankine cycle on board a diesel-electric railcar
}

Johan Peralez, Paolino Tona, Madiha Nadri, P. Dufour, Antonio Sciarretta

\section{To cite this version:}

Johan Peralez, Paolino Tona, Madiha Nadri, P. Dufour, Antonio Sciarretta. Optimal control for an organic rankine cycle on board a diesel-electric railcar. Journal of Process Control, 2015, 33, pp.1-15. 10.1016/j.jprocont.2015.03.009 . hal-01205902

\section{HAL Id: hal-01205902 \\ https://hal.science/hal-01205902}

Submitted on 28 Sep 2015

HAL is a multi-disciplinary open access archive for the deposit and dissemination of scientific research documents, whether they are published or not. The documents may come from teaching and research institutions in France or abroad, or from public or private research centers.
L'archive ouverte pluridisciplinaire $\mathbf{H A L}$, est destinée au dépôt et à la diffusion de documents scientifiques de niveau recherche, publiés ou non, émanant des établissements d'enseignement et de recherche français ou étrangers, des laboratoires publics ou privés. 


\title{
Optimal Control for an Organic Rankine Cycle on board a Diesel-Electric Railcar
}

\author{
J. Peralez ${ }^{\mathrm{a}, \mathrm{b}}$, P. Tona ${ }^{\mathrm{a}}$, M. Nadri ${ }^{\mathrm{b}}$, P. Dufour ${ }^{\mathrm{b}}$, A. Sciarretta ${ }^{\mathrm{c}}$ \\ ${ }^{a}$ IFP Energies nouvelles - Control, Signal and System Department \\ Rond-point de l'échangeur de Solaize, BP 369360 Solaize, France \\ (e-mail: \{johan.peralez, paolino.tona\}@ifpen.fr) \\ ${ }^{b}$ Université de Lyon, F-69003, France - Université Lyon 1 - CNRS UMR 5007 - Laboratory of Process Control \\ and Chemical Engineering (LAGEP) - 43 bd du 11 novembre, 69100 Villeurbanne, France \\ (e-mail: \{dufour, nadri\}@lagep.univ-lyon1.fr) \\ ${ }^{c}$ IFP Energies nouvelles - Control, Signal and System Department \\ 184 , avenue de Bois-Préau 92852 Rueil-Malmaison Cedex, France \\ (e-mail: antonio.sciarretta@ifpen.fr)
}

\begin{abstract}
This paper addresses the problem of maximizing the power produced by an organic rankine cycle (ORC) waste heat recovery system on board a diesel-electric railcar. A simplified model of the system allows the formulation of an optimal control problem that can be solved via dynamic programming (DP). To increase the smoothness and the accuracy of the solution obtained offline using the implementation of DP known as level-set DP, an improved version is developed, making use of adaptive grids for discretization. The analysis of the resulting optimal trajectory for the ORC control problem provides useful insight for both control design and system design. Based on these results, the optimal control problem is reformulated allowing online implementation via dynamic real-time optimization. The proposed approach is validated on a realistic simulator, showing significant benefits in the amount of recovered energy when compared with the classical, quasi-static approach found in ORC literature.
\end{abstract}

Keywords: Dynamic programming, backward reachability, real-time optimization, waste heat recovery, Rankine cycle, vehicle energy management.

\section{Introduction}

With new technologies continuously being introduced to further reduce vehicle fuel consumption, new control problems arise that must be efficiently solved to make the most of the innovation potential. This is for instance the case of engine waste heat recovery (WHR) systems based on the Rankine thermodynamic cycle. Applications of this technology exist, at different levels of maturity, for cars, trucks, trains and ships (see Sprouse and Depcik [27] for a recent overview). For some applications, such as WHR via organic rankine cycle (ORC) from Diesel engines of long-haul trucks, very interesting fuel consumption reductions have been reported (up to 6\%, according to Stanton [28]). As shown for instance in Peralez et al. [21], Horst et al. [12] and Xie and Yang [32], control of Rankine-based WHR systems for vehicular applications is far from being trivial.

In these applications, contrary to conventional (stationary) Rankine cycles, hot source dynamics depends on driving conditions and acts as a (highly-transient) time-varying disturbance. In 
this context, an effective control system is essential to attain satisfactory performance over a broad range of operating conditions, where "satisfactory" means safe, first of all, but also energetically efficient. In the still scarce literature on control design for Rankine WHR (see for instance the comprehensive overview presented in [27]), experimentally-validated strategies proposed so far for transport-related applications (such as Endo et al. [8], Peralez et al. [20]) focus on low-level control to ensure safe operation.

Few papers address supervisory control of Rankine WHR in the framework of integrated powertrain energy and emission management. The solution based on the Pontryagin minimum principle (PMP) presented in Merz et al. [18] includes a very simple dynamic Rankine model but cannot be implemented on line as such, while the heuristic strategy, also inspired by PMP, proposed in Willems, Frank et al. [30] does not use any explicit WHR model in the controller. A few other studies in ORC literature address the optimization of an economic objective, mostly using quasisteady-state models. Among them, Quoilin et al. [25, 26] focused on the optimal evaporating temperature in the case of an ORC with a volumetric expander, while Manente et al. [16]) considered an ORC with air-cooled condenser. In the field of ORC stabilization, Hou et al. [14], Zhang et al. [33] used a dynamic (linear) model to design a model predictive controller: a cost function associated to stabilization performance was then optimized.

On the other hand, several problems of transient control of energy production (see e.g Guzzella and Sciarretta [10]) on board vehicles in the presence of constraints have been addressed and solved via optimal control methods and in particular via dynamic programming (DP) (Bellman [3]).

However, to the authors' knowledge, no solution for online optimization of power production taking into account Rankine dynamics has been reported so far.

This paper addresses the problem of maximizing the power produced by an ORC WHR system on board a diesel-electric railcar. ${ }^{1}$ An experimentally-validated, reduced-order dynamic model allows the formulation of an optimal control problem that can be solved via DP. To increase the smoothness and the accuracy of the solution obtained using the state-of-the-art implementation of DP known as Level-Set DP (Elbert et al. [7]), a novel version of that algorithm, called AdaptiveGrid DP, is developed. The analysis of the resulting optimal trajectory for the ORC control problem provides useful insight for the design of an online control strategy, based on dynamic real-time optimization (DRTO, see e.g Würth et al. [31]).

This paper is organized as follows. Section 2 introduces the application and its specificities. A simplified model of the system and the formulation of the optimal control problem are proposed. Section 3 details the novel Adaptive-Grid DP algorithm — illustrated with a simple energy management example in Appendix A - providing an offline solution to the optimal control problem. Based on these results, the optimal control problem is reformulated in Section 4, allowing online implementation via DRTO. This approach is validated on a realistic simulator, showing significant increase in the amount of the energy recovered during a typical mission, when compared with the classical approach found in ORC literature, in which a quasi-static model is used for optimization.

\footnotetext{
${ }^{1}$ Part of this study, namely the offline approach, has been partially presented in the conference paper Peralez et al. [22]
} 


\section{Modeling and problem formulation}

\subsection{Problem statement}

The system under consideration is represented in Figure 1. It is a prototype ORC system for WHR from a diesel engine generating set ("Power Pack") manufactured by Alstom Transport for installation in diesel-electric railcars. A pump pressurizes an organic fluid (R365mfc) in liquid state and circulates it through a closed circuit at flow rate $\stackrel{*}{m}$. Via a by-pass valve, a fraction $V_{o, e}$ of engine exhaust gas is fed to a heat exchanger (evaporator) which transfers gas heat to the working fluid. The vaporized fluid then enters a turbine, which converts some of the kinetic energy into work, driving a generator connected to the Power Pack electrical network. Lastly, the fluid is recondensed before returning to the pump at low pressure. Cooling is provided by a fan with controllable air flow $\stackrel{*}{m}_{A}$. Notice that, although cooling is rarely taken into account in ORC optimization problems, it represents a significant cost in energy balances for ORC applications on board vehicles (Horst et al. [13]).

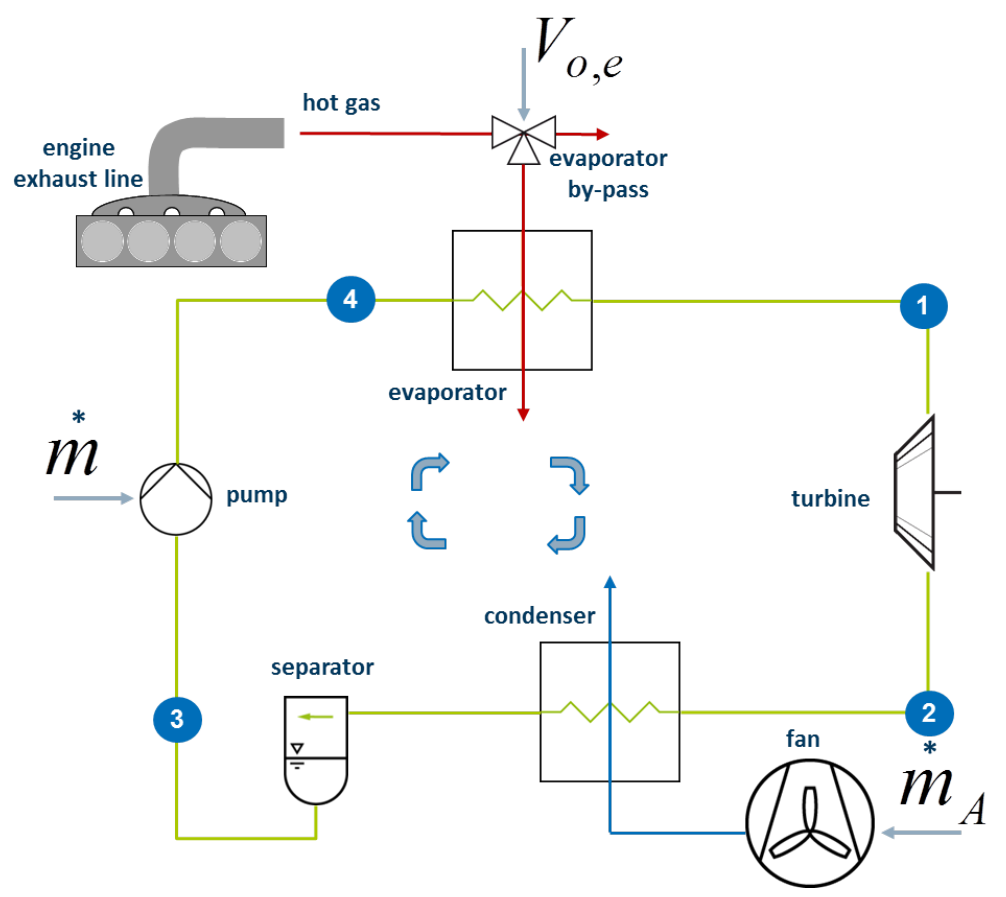

Figure 1: ORC system under investigation. Three control inputs are available: pump mass flow $\stackrel{*}{m}$, air mass flow $\stackrel{*}{m}_{A}$ and evaporator by-pass position $V_{o, e}$.

As the ratio between the power consumed by the auxiliaries and the power used for traction is relatively large on board trains (which benefit from a low aerodynamic friction and from a low rolling resistance coefficient), it can be safely assumed that all the power produced by the WHR system is fed to the electrical network. Consequently, the objective here is to find the control strategy that maximizes the energy $J$ recovered by the WHR system along a given mission of duration $t_{f}$ :

$$
J=\int_{0}^{t_{f}} P_{n e t}(t) d t
$$


where $P_{n e t}$ is given by the power produced by the turbine, $P_{\text {turb }}$, minus the powers consumed by the pump and the cooling system, $P_{\text {pump }}$ and $P_{A}$,

$$
P_{\text {net }}(t)=P_{\text {turb }}(t)-P_{\text {pump }}(t)-P_{A}(t)
$$

The mission profile considered herein, a portion of a typical intercity train trip, results in a set of external conditions for the ORC system, namely for the engine exhaust gas temperature $T_{\text {exh }}(t)$ and mass-flow $\stackrel{*}{m}_{\text {exh }}(t)$. A large variation of ambient temperature $T_{A}(t)$ is also considered as shown in Figure 2.

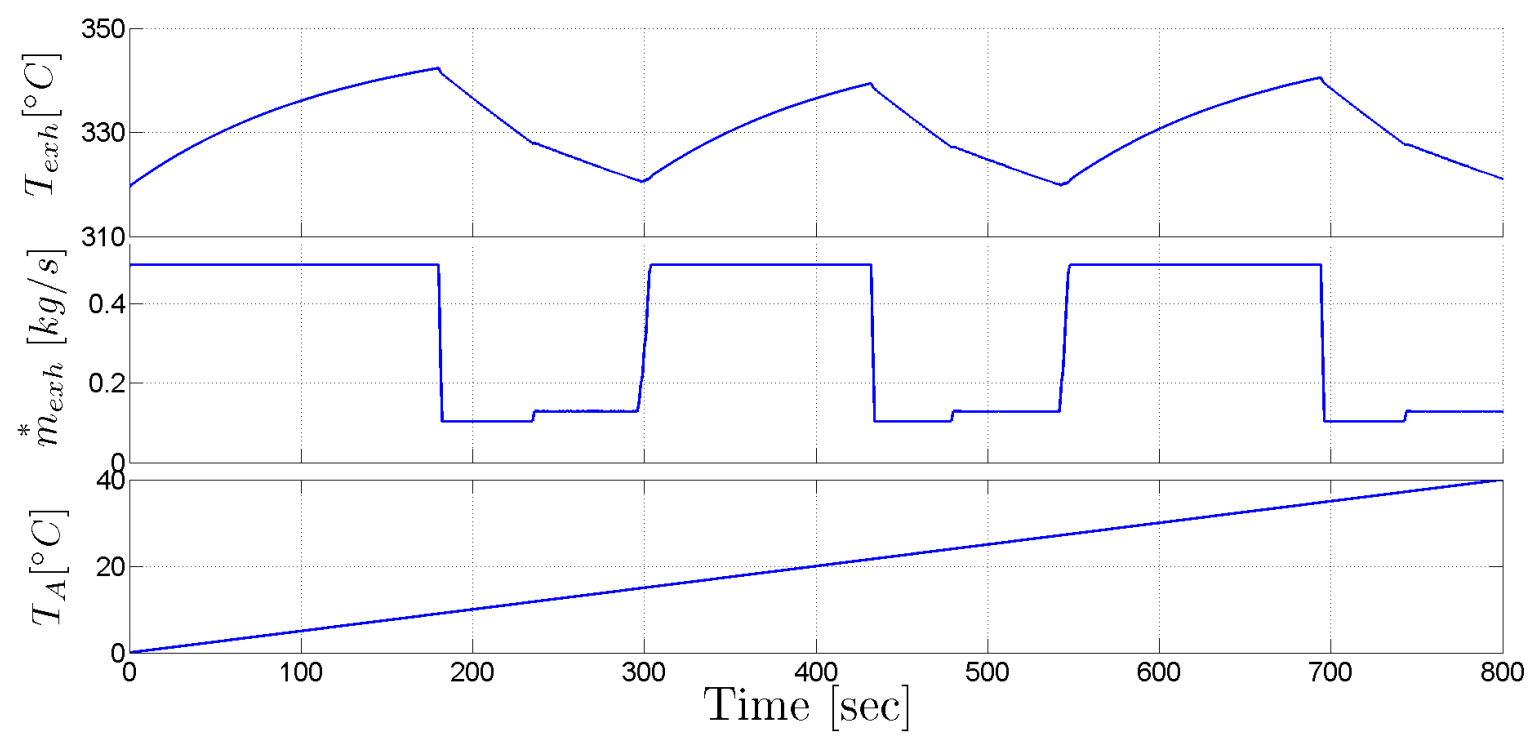

Figure 2: Input disturbances for ORC problem.

To solve this problem, a model is needed to predict the evolution of the variables in the expressions of powers (2). Model dimension should be small so as not to be confronted with the well-known "curse of dimensionality" (Powell [24]). The so-called moving boundaries method (see e.g. McKinley and Alleyne [17]) allows a realistic dynamic representation of heat exchangers with a limited number of state variables. Cheng et al. [5] proposes a model that considers an average wall temperature, while Peralez et al. [20] shows experimentally that, for an ORC, the wall temperatures have the slowest dynamics. Thus, it is possible to neglect the (much faster) fluid dynamics, thereby reducing the number of state variables.

In the following subsection, the thermodynamic cycle is first explained and described by mathematical expressions, then these dependencies are reduced by assumptions and simplifications, resulting in an implicit dynamic model.

\subsection{Two-state ORC model}

The thermodynamic cycle of the working fluid is represented in Figure 3. In the following, subscripts 1 to 4 will refer to the working fluid at each component outlet, namely the evaporator, the turbine, the condenser and the pump. 
Recoverable mechanical power from the turbine is usually expressed (Bao and Zhao [2]) in terms of a constant mechanical efficiency $\eta_{\text {turb }}$, of the working fluid mass-flow $\stackrel{*}{m}$ and of the enthalpy gradient $h_{2}-h_{1}$ by

$$
P_{\text {turb }}=\stackrel{*}{m}\left(h_{2}-h_{1}\right) \eta_{\text {turb }}
$$

Similarly, the efficiency $\eta_{\text {pump }}$ can be considered constant and the power consumed by the pump can be written (Borsukiewicz-Gozdur [4]) in terms of the pressure gradient $p_{1}-p_{3}$ and the fluid density $\rho_{3}$ as following

$$
P_{\text {pump }}=\stackrel{*}{m} \frac{p_{1}-p_{3}}{\rho_{3} \eta_{\text {pump }}} .
$$

The power consumed by the condenser fan can be considered proportional to the delivered air flow $\stackrel{*}{m}_{A}$ (Manente et al. [16]) and is given by

$$
P_{A}=\stackrel{*}{m}_{A} k_{A},
$$

where $k_{A}$ is the specific power consumption of the condenser fan.

Considering the thermodynamic cycle shown in Figure 3, fluid superheating at evaporator outlet (point 1) is assumed to be perfectly regulated at a constant value by the pump mass-flow. The fluid at the pump inlet (point 3) is assumed to be in the saturated liquid state. The slow dynamics of the system, i.e. the thermal dynamics of exchangers walls, are taken into account while the fluid is considered to be in equilibrium.

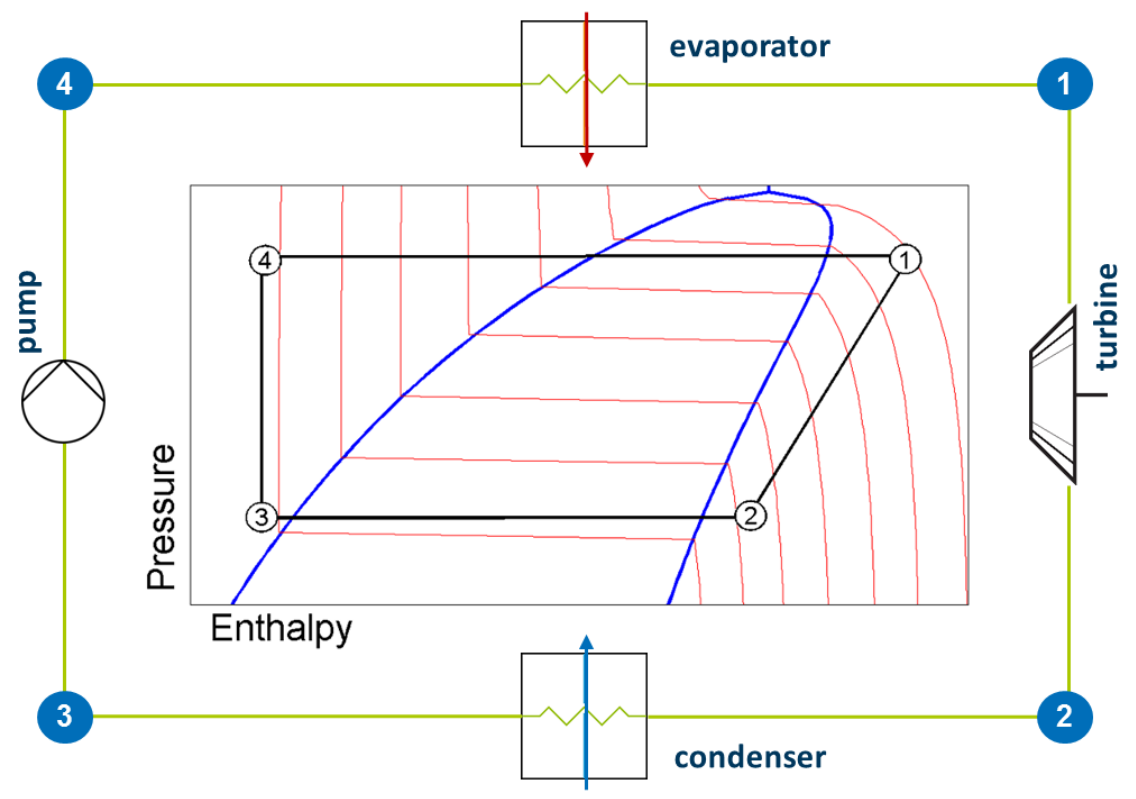

Figure 3: Rankine cycle for a dry fluid

Let $\bar{T}_{w, e}$ and $\bar{T}_{w, c}$ the average wall temperatures, $\bar{T}_{f, e}$ and $\bar{T}_{f, c}$ the average temperatures of the working fluid, $\bar{\alpha}_{f, e}$ and $\bar{\alpha}_{f, c}$, the average heat exchange coefficients between wall and fluid, where 
the subscripts $e$ and $c$ refer respectively to the evaporator and the condenser. The wall energy balance can be written as:

$$
\begin{aligned}
& M_{w, e} \dot{\bar{T}}_{w, e}=S_{f, e} \bar{\alpha}_{f, e}\left(\bar{T}_{f, e}-\bar{T}_{w, e}\right)+V_{o, e} \eta_{e x h} \stackrel{*}{m}_{e x h} c_{p, e x h}\left(T_{e x h}-\bar{T}_{w, e}\right) \\
& M_{w, c} \dot{\bar{T}}_{w, c}=S_{f, c} \bar{\alpha}_{f, c}\left(\bar{T}_{f, c}-\bar{T}_{w, c}\right)+\eta_{A} \stackrel{*}{m}_{A} c_{p, A}\left(T_{A}-\bar{T}_{w, c}\right),
\end{aligned}
$$

where $M_{w}$ is an equivalent thermal capacity, $S_{f}$ the wall-fluid exchange area, $S_{A}$ the wall-air exchange area and $\eta_{A}=1-\exp \left(-\frac{\alpha_{A} S_{A}}{\stackrel{*}{m}_{A} c_{p, A}}\right)$ reflects the decrease in heat exchange efficiency for large air mass flows (McKinley and Alleyne [17]), whereas $\eta_{\text {exh }}$ can be considered constant because of the lower mass flow involved.

In the following, $\bar{T}_{f, e}$ and $\bar{T}_{f, c}$ will be assumed equal to the evaporation temperatures respectively of the high-pressure and of the low-pressure sides of the ORC. $\bar{T}_{f, e}$ (resp. $\left.\bar{T}_{f, c}\right)$ is then an unimodal function of $p_{1}$ (resp. of $p_{3}$ ). Likewise, $h_{1}$ and $\rho_{1}$ depend only on $p_{1}$, while $h_{3}$ and $\rho_{3}$ depend on $p_{3}$.

Fluid energy equilibrium in the exchangers yields:

$$
\left\{\begin{array}{c}
0=h_{1}-S_{f, e} \bar{\alpha}_{f, e} \frac{\bar{T}_{w, e}-\bar{T}_{f, e}}{*}-h_{3} \\
0=h_{3}-S_{f, c} \bar{\alpha}_{f, c} \frac{\bar{T}_{w, c}-\bar{T}_{f, c}}{\stackrel{*}{m}}-h_{2},
\end{array}\right.
$$

where the mass flow $\stackrel{*}{m}$ is considered homogeneous throughout the circuit. Assuming supersonic flow condition, the pressure at turbine outlet is neglected (Feru et al. [9]) and the mass flow can be expressed as follows:

$$
\stackrel{*}{m}=c_{d} S \sqrt{2 \rho_{1} p_{1}}
$$

where $c_{d}$ is a (constant) discharge coefficient, and $S$ the equivalent section of the turbine nozzles.

Assuming a constant isentropic efficiency $\eta_{i s}$ of the turbine

$$
h_{2}=h_{1}-\eta_{i s}\left(h_{1}-h_{i s, 2}\right) \text {, }
$$

where $h_{i s, 2}$ corresponds to the enthalpy whose entropy is equal to that of point 1 and pressure is equal to that of point 3 . $h_{i s, 2}$ then depends on $p_{1}$ and $p_{3}$.

Using the previous expressions for $\stackrel{*}{m}$ and $h_{2}$, system $(7)$ can be written in the following implicit form:

$$
0=\varphi\left(\bar{T}_{w, e}, \bar{T}_{w, c}, p_{1}, p_{3}\right) .
$$

At each instant, from the knowledge of state variables $\bar{T}_{w, e}$ and $\bar{T}_{w, c}$, the implicit equation (10) can be solved numerically by iterating over $p_{1}$ and $p_{3}$, as illustrated in Figure 4 . Through the computation of fluid properties the expression of wall thermal dynamics can be found (6), as well as other key variables (such as $\stackrel{*}{m}$ in Figure 4 ).

The next subsection discusses model validation for transient operating conditions. 

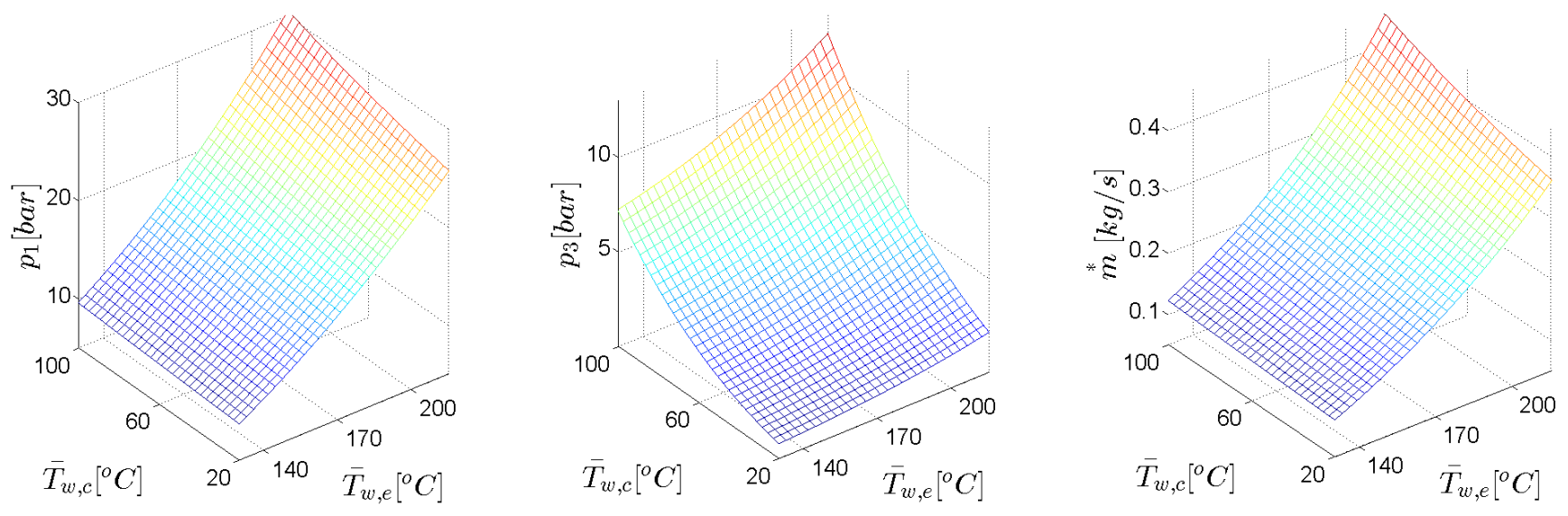

Figure $4: p_{1}, p_{3}$ and $\stackrel{*}{m}$ maps, function of $\bar{T}_{w, e}$ and $\bar{T}_{w, c}$

\subsection{Experimental validation of the reduced model}

In order to validate the single-state model of the heat-exchanger, the evaporator model response to transient external conditions is compared to experimental data. Transient conditions of exhaust gas entering the evaporator are shown in Figure 5(a).

The numerical value for the average heat exchange coefficient $\bar{\alpha}_{f, e}$ has been fitted against the pump mass flow $\stackrel{*}{m}$ experimental data.

Figure 5(b) compares model predictions of key working fluid variables at evaporator outlet, namely the mass flow $\stackrel{*}{m}$, the pressure $p_{1}$ and the temperature $T_{1}$, with the corresponding measurements. Relative average deviations are found to be about $3.3 \%$ for working fluid mass flow, $2 \%$ for pressure and less than $1 \%$ for temperature. This accuracy, demonstrated in transient conditions, motivates the use of the simplified model for a dynamic optimization purpose.

\subsection{Optimal control formulation for ORC-based WHR on board diesel-electric railcars}

Considering the mathematical model of an ORC described above, the formulation of the problem stated in Section 2.1 for WHR on board diesel-electric railcars will be developed in the following.

The aim is to find a control strategy that maximizes the energy recovered $J$, defined by equations (2)-(5), along a given mission of duration $t_{f}$. The cost functional $J$ to be maximized depends on time, control inputs $u=\left\{V_{o, e}, \stackrel{*}{m}_{A}\right\}$ and state vector $x=\left\{\bar{T}_{w, e}, \bar{T}_{w, c}\right\}$ whose dynamics are defined by the two-state ORC model equations (6)-(10). This maximization problem must be solved under the following safety constraint on pressure $p_{1}$ whereas bounds on the control inputs are set by technical considerations: the fraction of exhaust gas $\left(u_{1}\right)$ is bounded by $[0,1]$, whereas the air mass flow provided by the fan $\left(u_{2}\right)$ is between 0 and $4 \mathrm{~kg} \mathrm{~s}^{-1}$.

Then, the optimal control problem of the ORC can be formulated under the following minimization form:

$$
\min _{u(t)} \int_{0}^{t_{f}}-P_{\text {turb }}(x)+P_{\text {pump }}(x)+P_{A}(u) d t
$$



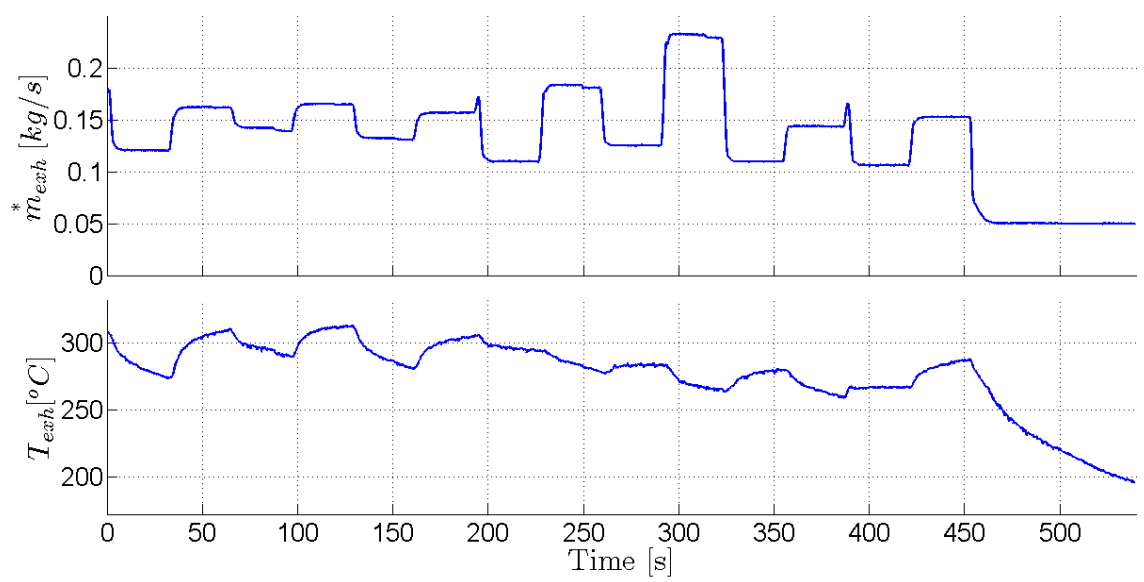

(a) External conditions.

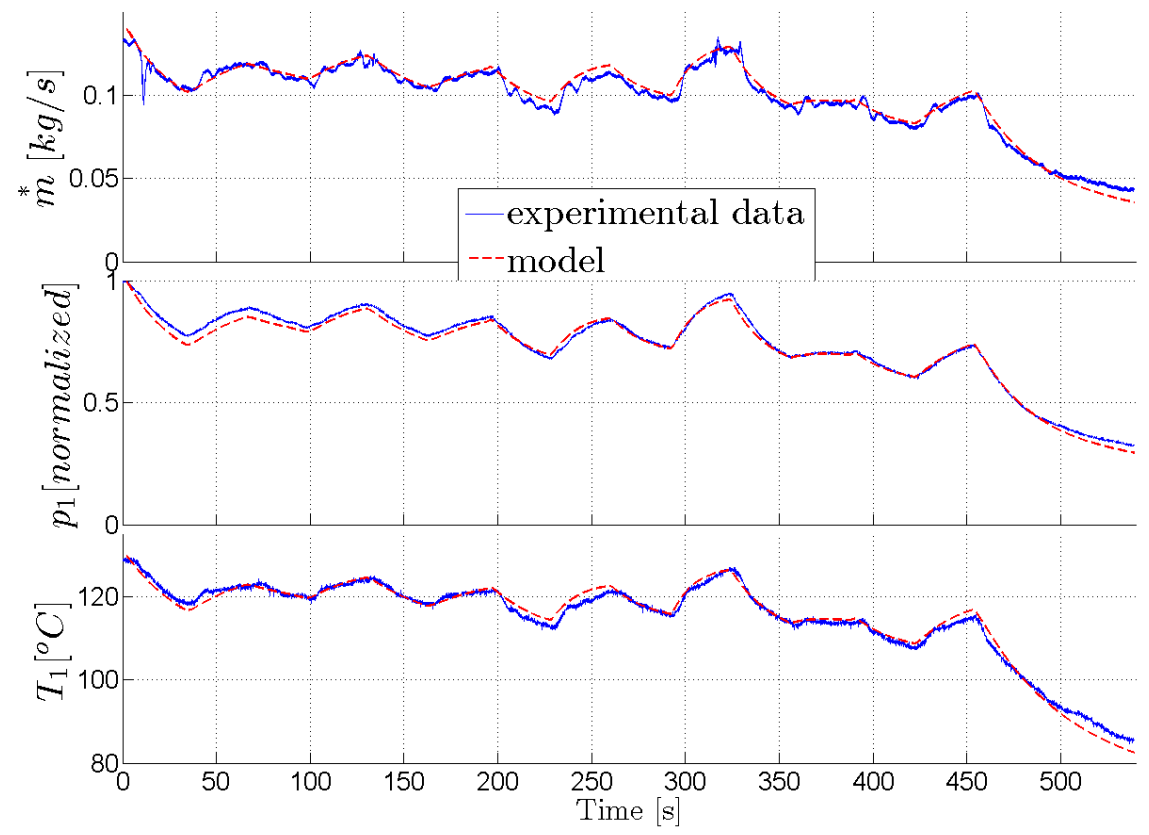

(b) Model prediction and measurement.

Figure 5: Single-state evaporator model: experimental validation

such that

$$
\begin{gathered}
\dot{x}=\left(\begin{array}{c}
S_{f, e} \bar{\alpha}_{f, e}\left(\bar{T}_{f, e}-x_{1}\right) / M_{w, e}+u_{1} \eta_{e x h} \stackrel{*}{m}_{e x h} c_{p, e x h}\left(T_{e x h}-x_{1}\right) / M_{w, e} \\
S_{f, c} \bar{\alpha}_{f, c}\left(\bar{T}_{f, c}-x_{2}\right) / M_{w, c}+\left(1-\exp \left(-\frac{\alpha_{A} S_{A}}{u_{2} c_{p, A}}\right)\right) u_{2} c_{p, A}\left(T_{A}-\bar{T}_{w, c}\right) / M_{w, c}
\end{array}\right), \\
x(0)=x_{0},
\end{gathered}
$$




$$
\left\{\begin{array}{c}
0=h_{1}-S_{f, e} \bar{\alpha}_{f, e} \frac{\bar{T}_{w, e}-\bar{T}_{f, e}}{\stackrel{*}{m}^{*}}-h_{3} \\
0=h_{3}-S_{f, c} \bar{\alpha}_{f, c} \frac{\bar{T}_{w, c}-\bar{T}_{f, c}}{m}-h_{2}, \\
p_{1}(x(t)) \leq 25 \mathrm{bar}, \forall t \in\left[0, t_{f}\right], \\
u(t) \in[0,1] \times[0,4], \forall t \in\left[0, t_{f}\right] .
\end{array}\right.
$$

Remark 1. Pump mass flow $\stackrel{*}{m}$ is not included as a decision variable in the above formulation as it is used to maintain superheating at a constant value. Indeed, it is usually accepted that, in ORCs, superheating should be maintained as low as possible in order to maximize cycle efficiency (Sprouse and Depcik [27], Bao and Zhao [2]). Based on two-time-scale considerations (working fluid dynamics is much faster than wall thermal dynamics), superheating is assumed perfectly regulated by pump mass flow (Peralez et al. [20] showed experimentally that such assumption is realistic, even in transient driving conditions).

\section{Offline dynamic optimization}

The main advantage of DP above other optimization methods is that it gives sufficient conditions for global optimality. Its main drawback is that the computational load grows exponentially with the number of state variables and control inputs (Powell [24], Lee and Wong [15]). DP is usually applied writing the system dynamics in a discrete form and the accuracy of the solution increases with the number of points considered. With the basic implementation, the necessary computational effort rapidly becomes untenable, especially in the presence of active state constraints as in the ORC problem.

In this section, the dynamic programming principle and the state-of-the-art implementation of DP known as Level-Set DP are first recalled. Then, in order to increase the smoothness and the accuracy of the solution while keeping an acceptable computational load, a novel version of that algorithm, called Adaptive-Grid DP, is developed. The solution obtained for the ORC optimal control problem is then analyzed, in order to get insight for the online strategy developed in Section 4 .

\subsection{Level-set Dynamic Programming principle}

\subsubsection{Dynamic Programming principle}

The generic optimal control problem to solve for a dynamic system with $n$ state variables and $m$ control inputs can be written as

$$
\begin{aligned}
\min _{u(t)} J(u(t)) & \\
\text { s.t. } & \\
\dot{x}(t) & =f(x(t), u(t), t) \\
x(0) & =x_{0} \\
x(t) & =\mathcal{X}(t) \subseteq \mathbb{R}^{n} \\
u(t) & =\mathcal{U}(t) \subseteq \mathbb{R}^{m}
\end{aligned}
$$


where

$$
J(u(t))=g\left(x\left(t_{f}\right)\right)+\int_{0}^{t_{f}} h(x(t), u(t), t) d t
$$

is the cost functional.

Since dynamic programming is a numerical algorithm, discretization of time, state space, and control space is required. Let the discrete-time model be

$$
x_{k+1}=f_{k}\left(x_{k}, u_{k}\right), k=0,1, \ldots, N-1
$$

where $x_{k} \in \mathcal{X}_{k}$ is the state vector and $u_{k} \in \mathcal{U}_{k}$ the control signal vector.

Based on the principle of optimality introduced by Bellman [3], DP proceeds backwards in time to evaluate the optimal cost-to-go function $\mathcal{J}_{k}\left(x^{i}\right)$ at every node $x^{i}$ of the discretized time-state space Sundström et al. [29]:

Step 1: Initialization

$$
\mathcal{J}_{N}\left(x^{i}\right)=\left\{\begin{array}{l}
g_{N}\left(x^{i}\right), \text { for } x^{i} \in \mathcal{X}_{N} \\
\infty \text { else. }
\end{array}\right.
$$

Following steps: Backward iteration for $k=N-1$ to 0

$$
\mathcal{J}_{k}\left(x^{i}\right)=\min _{u_{k} \in U_{k}}\left\{g_{k}\left(x^{i}, u_{k}\right)+\mathcal{J}_{k+1}\left(f_{k}\left(x^{i}, u_{k}\right)\right)\right\}
$$

The optimal control is the argument that minimizes the right-hand side of equation (25) for each $x^{i}$ at time index $k$, which yields the optimal control policy $\pi=\left\{u_{0}(x), u_{1}(x), \ldots, u_{N-1}(x)\right\}$. This map is used to find the optimal control signal during a forward simulation of model (23) starting from a given initial state $x_{0}$. Since the resulting state trajectory does not generally coincide with the nodes $x^{i}$, interpolation is used.

\subsubsection{Level-set algorithm}

A major issue to consider when implementing a DP algorithm, is the definition of the costto-go function for an infeasible state vector. Grid points that are not backward-reachable should have infinite cost as in (24). However, this causes numerical problems as illustrated in Figure 6. When running step $k$ of backward iteration, the cost-to-go $\mathcal{J}_{k+1}(x)$ is known for all grid points $x^{i}$. In order to evaluate the cost-to-go $\mathcal{J}_{k}\left(x^{p}\right)$, all possible control candidates $u \in \mathcal{U}_{k}$ are applied. In Figure 6, the point $f_{k}\left(x^{p}, u^{1}\right)$ is in the backward-reachable space. But since the value of $J_{k+1}\left(x^{i+1}\right)$ is infinite, interpolation will lead to consider that the cost-to-go of $x_{k}^{i+2}$ is infinite too.

The classical method to deal with this problem consists in using a large but finite value for an infeasible state vector. The value of this penalty represents a critical parameter that is difficult to calibrate. Nevertheless, the penalty method results in a steep gradient of the cost-to-go function near the boundary and cannot completely solve the aforementioned problem.

Another approach is based on the concept of backward-reachable space, as proposed in [1, 29] for single-input single-state systems. For larger systems, the level-set DP algorithm, introduced by Elbert et al. [7], evaluates the backward-reachable space in parallel with performance index evaluation. A level-set function $\mathcal{I}$ is considered that associates a real value to the state $x$ :

$$
\mathcal{I}: X \subseteq \mathbb{R}^{n} \rightarrow \mathbb{R}
$$




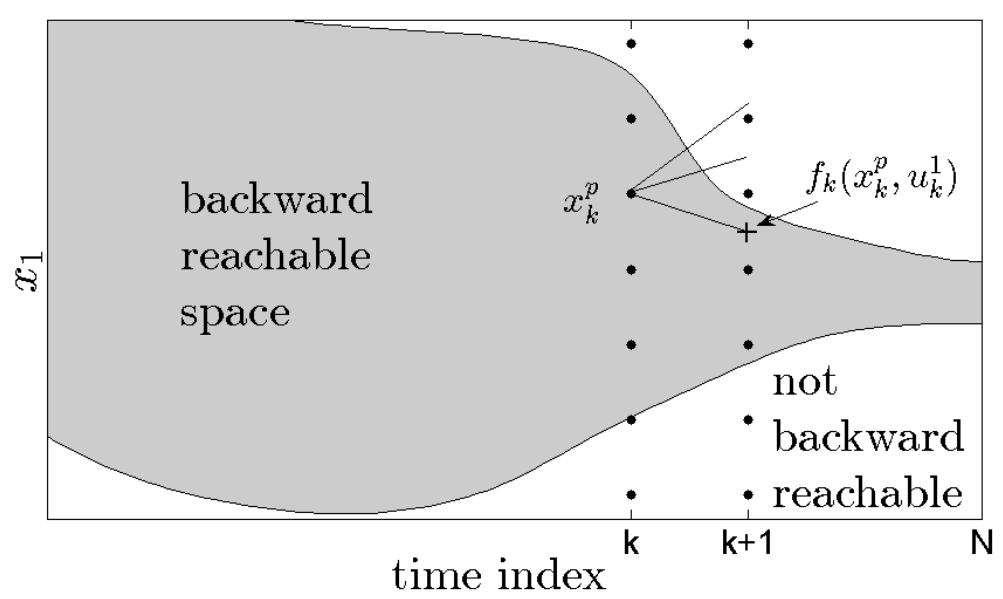

Figure 6: Numerical issues near boundary surface.

where $\mathcal{I}(x) \leq 0$ if $x$ is in reachable space and $\mathcal{I}(x)>0$ else. Such a function can represent nonconvex regions, moreover, the number of state variables and control inputs is not limited. The level-set function is evaluated for grid points $x^{i}$ at each time index $k$ yielding a grid evaluation $\mathcal{I}_{x}^{k}$. At the next backward iteration step, interpolation is used to determine whether a point $x$ is backward reachable or not. It is assumed that the final state constraint is given as a target set $T$, which is defined by a level-set function $h(x)$

$$
\begin{aligned}
h: X_{N} & \rightarrow \mathbb{R}, \text { where } X_{N} \subseteq \mathbb{R}^{n} \\
T & =\left\{x \in X_{N} \mid h(x) \leq 0\right\} .
\end{aligned}
$$

Then the DP principle is applied, where the cost-to-go function to minimize is the level-set function $\mathcal{I}$ :

Step 1: Initialization

$$
\mathcal{I}_{N}\left(x^{i}\right)=h\left(x^{i}\right)
$$

Following steps: Backward iteration for $k=N-1$ to 0

$$
\mathcal{I}_{k}\left(x^{i}\right)=\min _{u_{k} \in U_{k}}\left\{\mathcal{I}_{k+1}\left(f_{k}\left(x^{i}, u_{k}\right)\right)\right\}
$$

\subsection{A new adaptive-grid DP algorithm}

At the expense of an acceptable increase of computational load, the level-set DP algorithm introduced in Elbert et al. [7] can be modified to provide a smoother and more accurate solution. Differences with original algorithm consist in the second discretization step of state space in the backward algorithm, defined by (33)-(37), and in the finer discretization of control space in the forward algorithm, defined by (44).

\subsubsection{Adaptive-grid DP backward algorithm}

To improve the accuracy of the solution, the backward algorithm introduced in Elbert et al. $[7]$ is modified as follows. 
Step 1: Initialize $\mathrm{k}=\mathrm{N}$ and the level-set and cost-to-go functions as:

$$
\begin{array}{r}
I_{N}\left(x^{i}\right)=h\left(x^{i}\right) \\
J_{N}\left(x^{i}\right)=g_{N}\left(x^{i}\right) .
\end{array}
$$

Considering that $x^{i} \in \mathbb{R}^{n}$ let $x^{i}=\left[\begin{array}{lll}x^{i}(1) & \ldots & x^{i}(n)\end{array}\right]$. A second discretization of state space $\tilde{X}_{N}$ is introduced:

$$
\tilde{X}_{N}=\left\{\tilde{x}_{N}^{1}, \tilde{x}_{N}^{2}, \ldots, \tilde{x}_{N}^{q}\right\}
$$

where each $j^{\text {th }}$ component of vector $\tilde{x}_{N}^{i}$ is bounded below by $\underline{x}_{N}(j)$ and above by $\bar{x}_{N}(j)$ as follows:

$$
\begin{aligned}
& \underline{x}_{N}(j)=\min _{i \in\{1, \ldots, q\}}\left\{x_{N}^{i}(j) \mid 0<\mathcal{I}_{N}\left(x_{N}^{i}\right)\right\} \\
& \bar{x}_{N}(j)=\max _{i \in\{1, \ldots, q\}}\left\{x_{N}^{i}(j) \mid 0<\mathcal{I}_{N}\left(x_{N}^{i}\right)\right\}
\end{aligned}
$$

As illustrated in Figure 7 this second discretization may improve the accuracy of level-set and cost-to-go estimations:

$$
\begin{array}{r}
\tilde{I}_{N}\left(\tilde{x}^{i}\right)=h\left(\tilde{x}^{i}\right) \\
\tilde{J}_{N}\left(\tilde{x}^{i}\right)=g_{N}\left(\tilde{x}^{i}\right) .
\end{array}
$$

Step 2: Reduce $k$ by 1 and update the level-set function by

$$
I_{k}\left(x^{i}\right)=\min _{u_{k} \in U_{k}}\left\{\tilde{I}_{k+1}\left(f_{k}\left(x^{i}, u_{k}\right)\right)\right\} .
$$

A second discretization $\tilde{X}_{k}$ is computed in the same way as for the first step. Then the level-set function is updated by

$$
\tilde{I}_{k}\left(\tilde{x}^{i}\right)=\min _{u_{k} \in U_{k}}\left\{\tilde{I}_{k+1}\left(f_{k}\left(\tilde{x}^{i}, u_{k}\right)\right)\right\} .
$$

Step 3: For each grid point $\tilde{x}^{i}$, find the set of control signals for which the system trajectory ends up inside the backward-reachable space at the next time step

$$
U_{k}^{F}\left(x^{i}\right)=\left\{u_{k} \in U_{k} \mid \tilde{I}_{k+1}\left(f_{k}\left(\tilde{x}^{i}, u_{k}\right)\right) \leq 0\right\},
$$

and the one control candidate that minimizes the level-set function

$$
{\stackrel{*}{u_{k}}}_{k}\left(\tilde{x}^{i}\right)=\arg \min _{u_{k} \in U_{k}}\left\{\tilde{I}_{k+1}\left(f_{k}\left(\tilde{x}^{i}, u_{k}\right)\right)\right\} .
$$


Step 4: Update the optimal cost-to-go by the following rule: if at least one valid control candidate is found, i.e., $U_{k}^{F}\left(x^{i}\right) \neq \emptyset$, then calculate the cost-to-go based upon the optimal candidate

$$
\tilde{J}_{k}\left(\tilde{x}^{i}\right)=\min _{u_{k} \in U_{k}^{F}\left(x^{i}\right)}\left\{g_{k}\left(\tilde{x}^{i}, u_{k}\right)+\tilde{J}_{k+1}\left(f_{k}\left(\tilde{x}^{i}, u_{k}\right)\right)\right\} .
$$

If, however, the grid point is not backward-reachable, then calculate the cost-to-go based on the control input $\stackrel{*}{u}_{k}\left(\tilde{x}^{i}\right)$

$$
\tilde{J}_{k}\left(\tilde{x}^{i}\right)=g_{k}\left(\tilde{x}^{i}, \stackrel{*}{u}_{k}\right)+\tilde{J}_{k+1}\left(f_{k}\left(\tilde{x}^{i}, \stackrel{*}{u}_{k}\right)\right)
$$

and repeat steps $2-4$ until $k=0$.
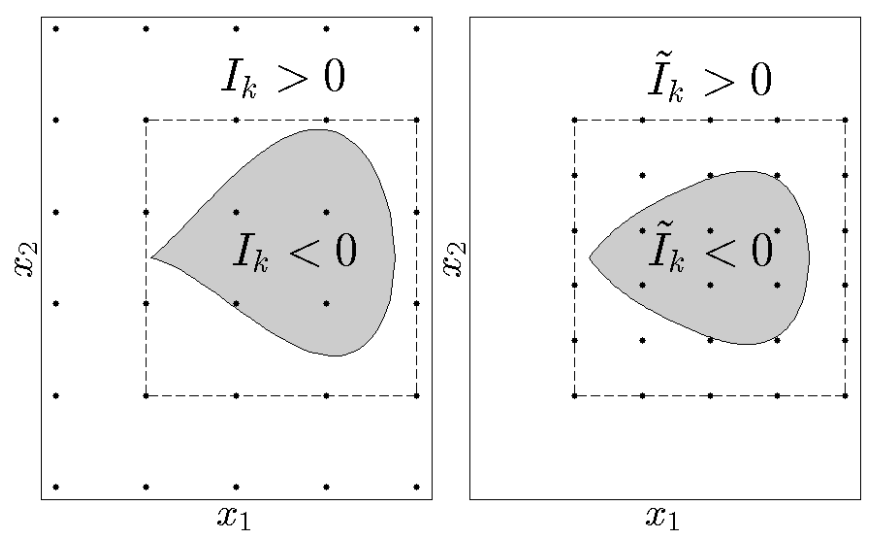

Figure 7: A first estimation $I_{k}$ of level-set function is computed on the whole state space (left plot). A second discretization (right plot) allows to improve the accuracy of both level-set and cost-to-go functions.

\subsubsection{Adaptive-grid DP forward algorithm}

To increase smoothness, the forward algorithm introduced in Elbert et al. [7] is used with a finer discretization of the control space:

$$
\tilde{U}_{k}=\{\underbrace{u_{k}^{1}, \ldots, u_{k}^{r}}_{U_{k}}, \ldots, u_{k}^{\tilde{r}}\}
$$

Step 1: Initialize $k=0$ and $x_{0}$ at initial condition.

Step 2: Increase $k$ by 1 and find the feasible control candidates

$$
\tilde{U}_{k}^{F}\left(x_{k}\right)=\left\{u_{k} \in \tilde{U}_{k} \mid \tilde{I}_{k+1}\left(f_{k}\left(x_{k}, u_{k}\right)\right) \leq 0\right\},
$$

Step 3: Find the optimal control input

$$
u_{k}^{0}\left(x_{k}\right)=\arg \min _{u_{k} \in \tilde{U}_{k}^{F}}\left\{g_{k}\left(x_{k}, u_{k}\right)+\tilde{J}_{k+1}\left(f_{k}\left(x_{k}, u_{k}\right)\right)\right\}
$$


Step 4: Simulate the system using the optimal control input

$$
x_{k+1}=f_{k}\left(x_{k}, u_{k}^{o}\right)
$$

and repeat steps $2-4$ until $k=N$.

Remark 2. Note that the computational effort involved in the finer discretization $\tilde{U}_{k}(44)$ is limited. Since steps 2 - 4 of the algorithm are applied to one value of $x_{k}$, the number of additional calls to the model is $N(\tilde{r}-r)$.

Before application to the ORC case study, the new algorithm has been validated on a simple energy management example, where an analytic solution exists. In this case, as shown in Appendix A, the results obtained with the adaptive grid DP are close to the analytic solution even with a coarse discretization.

\subsection{DP solution for ORC-based WHR on board diesel-electric railcars}

The ORC optimal control problem exposed in Section 2.4 is solved using the new adaptive-grid DP algorithm with numbers of grid points set to $N=N_{x 1}=N_{x 2}=N_{u 1}=N_{u 2}=21$ and with a sampling time set to 1 second. Figure 8 shows the system trajectory obtained. High and low pressures (respectively in the evaporator and in the condenser) periodically rise in response to the increase in the exhaust gas mass flow from the mission profile (Figure 2). In response, evaporator by-pass is open up to limit the high pressure at the safety constraint (15). On the other hand, the second control input exhibits a noncausal behavior: for instance, it can be observed that a few seconds before the external condition $\stackrel{*}{m}$ exh rises, $\stackrel{*}{m}$ A decreases at first. Such a behavior can be explained considering that the efficiency of heat exchange with ambient air improves when condenser wall temperature (and consequently pressure $p_{3}$ ) is higher.

In order to evaluate the benefits of the new algorithm, the ORC optimal control problem was also solved with the original level-set DP algorithm. It was found that the number of grid points $N=N_{x 1}=N_{x 2}=N_{u 1}=N_{u 2}=34$ was required to reach an equivalent accuracy. It resulted in a computational time about 4.5 times smaller for the adaptive grid DP. Despite the reduction in computational load obtained with the adaptive-grid algorithm, the execution times of the DP are still too long for online implementation. However, the DP solution remains very useful as an analysis tool to understand (optimal) ORC behavior. From the results above, one can infer that tracking control of condenser pressure can be obtained using the air mass flow $\stackrel{*}{m}_{A}$ to regulate condenser cooling, which would in turn ensure the pressure ratio required to run the turbine efficiently. On the other hand, the evaporator by-pass can be used to enforce safety limits in terms of pressure and temperature.

The DP solution also highlights the influence of ambient temperature on the overall efficiency of the ORC system. In fact, the power recovered by the turbine is substantially reduced for high ambient temperatures (during the end of the simulation), whereas the power consumed by the pump is scarcely affected.

These considerations will be used as design guidelines for the online supervisory control which is the object of section Section 4 . 


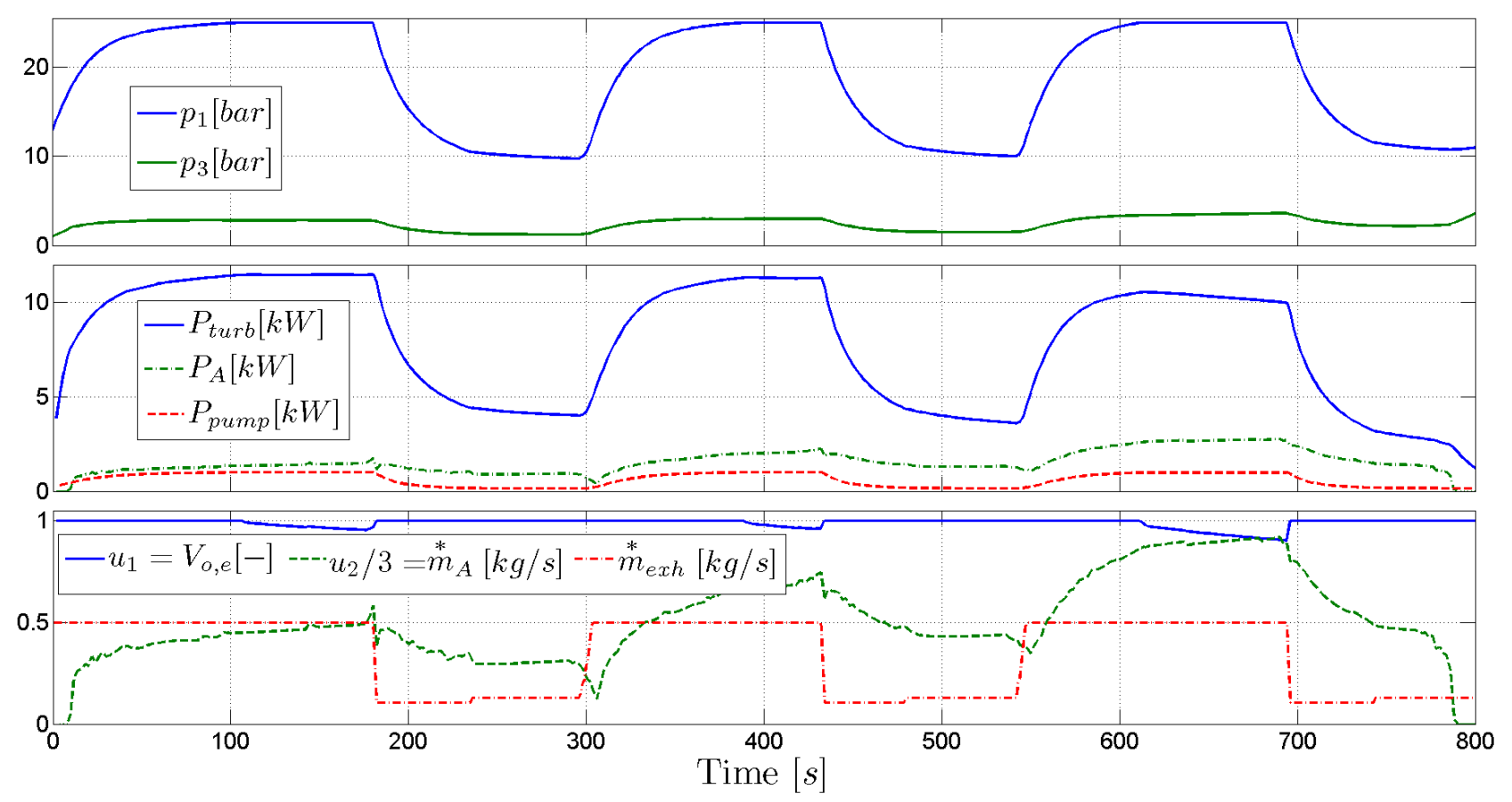

Figure 8: Adaptive-grid DP solution: time evolution of the ORC system characteristics

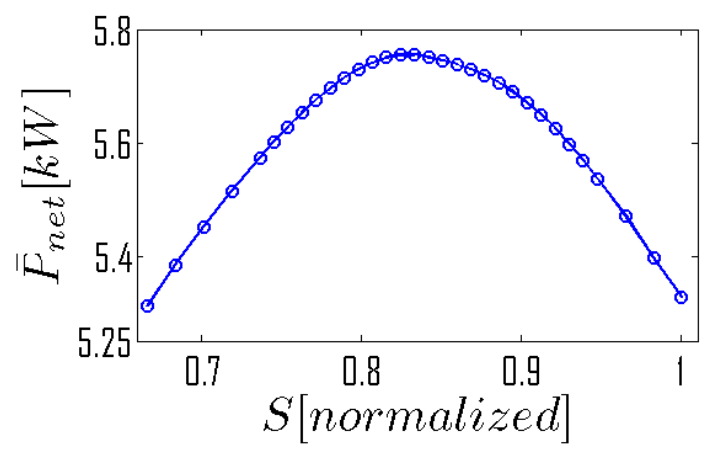

Figure 9: Influence of the equivalent turbine nozzle surface $S$ in term of mean net power recovered $\bar{P}_{\text {net }}$.

\subsection{Other uses of the DP approach}

Solving the optimal control problem introduced above can also prove useful at system design stage, especially considering that transient behavior is accounted for in system performance evaluation. The problem can be formulated for variants of the system under consideration, obtained with different sets of model parameters. Thanks to the good accuracy achievable by the adaptivegrid DP algorithm at low levels of discretization, it becomes feasible to study the impact of these (numerous) parameters within reasonable simulation times.

To illustrate this, the influence of a key design parameter - namely the equivalent turbine nozzle surface $S$ - on the ORC efficiency is investigated. The optimal control problem is then solved for different values of $S$ and the corresponding efficiencies are compared in term of mean net recovered power $\bar{P}_{\text {net }}$ (Figure 9). Such simulations help us to conclude that an optimal value 
for the surface $S$ may be found. Working fluid choice or exchangers sizing - taken into account in the two-state model described in Section 2.2 - are examples of other design parameters whose influence could be studied with this approach.

Since the proposed improvement does not rely on a specific structure, application of the new algorithm should also be beneficial to other optimal control problems. Yet, it has not been proved that the novel "adaptive-grid" algorithm consistently outperforms the original level-set algorithm in different contexts than those presented in this article.

\section{Online supervisory control}

One way to design an online supervisory control for the ORC is to directly solve the problem exposed in Section 2.4. Unfortunately, the limits of computational resources, particularly stringent in an embedded context, prevent achieving accurate real time solutions. Computational issues are mainly due to: i) the non-linearities of the model, ii) the implicit structure of the model, iii) the multi-input multi-output nature of the optimal control problem. Also, all disturbances need to be known over the whole cycle.

The aim of the following section is to investigate how to reformulate the problem in order to overcome these issues and find a real-time implementable (sub) optimal control strategy, regardless of the cycle considered. This is done in three steps. As a first step, through a new choice of dynamic state variables, it is shown that the ORC model can be written in an explicit form. This results in saving computation time. Moreover, the new state variables (the pressures $p_{1}$ and $p_{3}$ ) are directly measurable, avoiding observability issues. Then, insight given by the DP solution (Section 3.3) is used to simplify the optimal control problem formulation. In what follows, it is inferred that the evaporator by-pass can enforce the pressure (safety) limit and that the air mass flow should be used to optimize cycle efficiency. Finally, the main result is stated giving a new dynamic real-time optimization strategy.

\subsection{Explicit two-state ORC model}

To transform the implicit ORC model given by the system (6)-(10) into an ordinary differential equation (ODE) system of the following form

$$
\left\{\begin{aligned}
\dot{T}_{w} & =f\left(T_{w}, p, t\right) \\
\dot{p} & =-\left(\left.\frac{\partial \varphi}{\partial p}\right|_{\left(T_{w}, p\right)}\right)^{-1}\left(\left.\frac{\partial \varphi}{\partial T_{w}}\right|_{\left(T_{w}, p\right)}\right) f\left(T_{w}, p, t\right),
\end{aligned}\right.
$$

where $T_{w}=\left[\begin{array}{ll}\bar{T}_{w, e} & \bar{T}_{w, c}\end{array}\right]^{T}, p=\left[\begin{array}{ll}p_{1} & p_{3}\end{array}\right]^{T}$,

$$
\begin{gathered}
f=\left(\begin{array}{cc}
S_{f, e} \bar{\alpha}_{f, e}\left(\bar{T}_{f, e}-\bar{T}_{w, e}\right) / M_{w, e}+V_{o, e} \eta_{e x h} \stackrel{*}{m}_{e x h} c_{p, e x h}\left(T_{e x h}-\bar{T}_{w, e}\right) / M_{w, e} \\
S_{f, c} \bar{\alpha}_{f, c}\left(\bar{T}_{f, c}-\bar{T}_{w, c}\right) / M_{w, c}+\eta_{A} \stackrel{*}{m}_{A} c_{p, A}\left(T_{A}-\bar{T}_{w, c}\right) / M_{w, c},
\end{array}\right), \\
\frac{\partial \varphi}{\partial p}=\left(\begin{array}{cc}
-\left(h_{1}-h_{3}\right) \frac{\partial \stackrel{*}{\partial}}{\partial p_{1}}-\stackrel{*}{m} \frac{\partial h_{1}}{\partial p_{1}}-S_{f, e} \bar{\alpha}_{f, e} \frac{\partial \bar{T}_{f, e}}{\partial p_{1}} & \stackrel{* h_{3}}{\partial p_{3}} \\
\stackrel{*}{m} \frac{\partial h_{2}}{\partial p_{1}}-\frac{\partial \stackrel{*}{\partial}}{\partial p_{1}}\left(h_{3}-h_{2}\right) & \stackrel{*}{m}\left(\frac{\partial h_{2}}{\partial p_{3}}-\frac{\partial h_{3}}{\partial p_{3}}\right)-S_{f, c} \bar{\alpha}_{f, c} \frac{\partial \bar{T}_{f, c}}{\partial p_{3}}
\end{array}\right),
\end{gathered}
$$


and

$$
\frac{\partial \varphi}{\partial T_{w}}=\left(\begin{array}{cc}
S_{f, e} \bar{\alpha}_{f, e} & 0 \\
0 & S_{f, c} \bar{\alpha}_{f, c}
\end{array}\right)
$$

one has to check that $\frac{\partial \varphi}{\partial p}$ is of full rank (Hammouri and Nadri [11]).

Proposition 1. Let $\Omega$ be the set which represents the physical domain:

$$
\Omega=\left\{\left(T_{w}, p\right) \in \mathbb{R}^{2} \times \mathbb{R}^{2}, p_{1} \leq p_{3}, p_{1}<p^{c r i t}, p_{3}>0\right\},
$$

where $p^{\text {crit }}$ refers to the critical pressure for the considered working fluid. For every $\left(T_{w}, p\right) \in \Omega$, $\frac{\partial \varphi}{\partial p}$ is of full rank.

Proof 1. Consider the following notation

$$
\frac{\partial \varphi^{T}}{\partial p} \triangleq\left(\begin{array}{ll}
a & b \\
c & d
\end{array}\right)
$$

where

$$
\left\{\begin{aligned}
a & =-\left(h_{1}-h_{3}\right) \frac{\partial \stackrel{*}{\partial p_{1}}}{m}-\stackrel{*}{m} \frac{\partial h_{1}}{\partial p_{1}}-S_{f, e} \bar{\alpha}_{f, e} \frac{\partial \bar{T}_{f, e}}{\partial p_{1}} \\
b & =\stackrel{*}{m} \frac{\partial h_{2}}{\partial p_{1}}-\frac{\partial m}{\partial p_{1}}\left(h_{3}-h_{2}\right) \\
c & =\stackrel{*}{m} \frac{\partial h_{3}}{\partial p_{3}} \\
d & =\stackrel{*}{m}\left(\frac{\partial h_{2}}{\partial p_{3}}-\frac{\partial h_{3}}{\partial p_{3}}\right)-S_{f, c} \bar{\alpha}_{f, c} \frac{\partial \bar{T}_{f, c}}{\partial p_{3}} .
\end{aligned}\right.
$$

To show that $\frac{\partial \varphi}{\partial p}$ is well defined, it is sufficient to show that its transpose is strictly diagonally dominant, namely that $|a|>|b|$ and $|d|>|c|$.

Considering the thermodynamic cycle of Figure 3 and assuming that $\left(T_{w}, p\right) \in \Omega$, it can be deduced that

$$
|a|=\left(h_{1}-h_{3}\right) \frac{\partial \stackrel{*}{\partial p_{1}}}{m}+\stackrel{*}{m} \frac{\partial h_{1}}{\partial p_{1}}+S_{f, e} \bar{\alpha}_{f, e} \frac{\partial \bar{T}_{f, e}}{\partial p_{1}} .
$$

Indeed, $h_{1}$ and $\bar{T}_{f, e}$ (the saturation temperature) are increasing functions of $p_{1}$. Moreover, $h_{1}>h_{3}$ and the product $S_{f, e} \bar{\alpha}_{f, e}$ is positive. Finally, since the mass flow can be expressed as

$$
\stackrel{*}{m}=c_{d} S \sqrt{2 \rho_{1} p_{1}}
$$

it is deduced that $\stackrel{*}{m}$ is an increasing function of $p_{1}$.

Using the same steps as above,

$$
|b|=\stackrel{*}{m} \frac{\partial h_{2}}{\partial p_{1}}-\frac{\partial \stackrel{*}{m}}{\partial p_{1}}\left(h_{3}-h_{2}\right) .
$$


From (53) and (52), it yields

$$
|a|-|b|=\left(h_{1}-h_{2}\right) \frac{\partial \stackrel{*}{m}}{\partial p_{1}}+\stackrel{*}{m}\left(\frac{\partial h_{1}}{\partial p_{1}}-\frac{\partial h_{2}}{\partial p_{1}}\right)+S_{f, e} \bar{\alpha}_{f, e} \frac{\partial \bar{T}_{f, e}}{\partial p_{1}} .
$$

From Figure 3, the resultant work $\left(h_{1}-h_{2}\right)>0$ which may be recovered by the turbine is an increasing function of the high pressure $p_{1}$, i.e $\left(\frac{\partial h_{1}}{\partial p_{1}}-\frac{\partial h_{2}}{\partial p_{1}}\right)>0$. Then $|a|>|b|$.

Using the same approach, it comes

$$
|c|-|d|=-\stackrel{*}{m} \frac{\partial h_{2}}{\partial p_{3}}+S_{f, c} \bar{\alpha}_{f, c} \frac{\partial \bar{T}_{f, c}}{\partial p_{3}} .
$$

Using the expression of $\stackrel{*}{m}$, it comes that the following condition on system sizing is required

$$
\frac{S_{f, c}}{c_{d} S}<\stackrel{*}{m} \frac{\partial h_{2}}{\partial p_{3}}\left(\bar{\alpha}_{f, c} \frac{\partial \bar{T}_{f, c}}{\partial p_{3}}\right)^{-1} .
$$

In practice, this condition is realistic because the right-hand part of (54) evaluated in the physical domain is very large.

Consequently, the condition $|d|>|c|$ is verified.

This achieves the verification of Assumption1.

Now, considering system (7), $T_{w}$ can be deduced from $p_{1}$ and $p_{3}$

$$
T_{w}\left(p_{1}, p_{3}\right)=\left(\begin{array}{l}
\stackrel{*}{m} \frac{h_{1}-h_{3}}{S_{f, e} \bar{\alpha}_{f, e}}+\bar{T}_{f, e} \\
* \frac{h_{3}-h_{2}}{m} \frac{\bar{T}_{f, c}}{S_{f, c} \bar{\alpha}_{f, c}}
\end{array}\right) .
$$

Then, $T_{w}$ can be removed from the state vector and the ORC dynamics can be described by the following ODE equation:

$$
\dot{p}=-\left(\left.\frac{\partial \varphi}{\partial p}\right|_{\left(T_{w}, p\right)}\right)^{-1}\left(\left.\frac{\partial \varphi}{\partial T_{w}}\right|_{\left(T_{w}, p\right)}\right) f\left(T_{w}, p, t\right) .
$$

\subsection{Evaporator pressure control}

The original optimal problem with the two decision variables $\left\{V_{o, e}, \stackrel{*}{m}{ }_{A}\right\}$ will be split into two simpler sub-problems. First, a closed-loop controller for pressure $p_{1}$ acting on $V_{o, e}$ is designed. Then a dynamic real-time optimization strategy with $\stackrel{*}{m}$ as a decision variable is described.

In order to take into account safety limits, a closed-loop is built to regulate the high-pressure $p_{1}$. For this, the specific structure of dynamic model (56) will be exploited, exhibiting that it is affine in $V_{o, e}$, the evaporator bypass control input. 
Let

$$
\begin{aligned}
A & \triangleq-\left(\left.\frac{\partial \varphi}{\partial p}\right|_{\left(T_{w}, p\right)}\right)^{-1}\left(\left.\frac{\partial \varphi}{\partial T_{w}}\right|_{\left(T_{w}, p\right)}\right) \\
& \triangleq\left(\begin{array}{ll}
A_{11} & A_{12} \\
A_{21} & A_{22},
\end{array}\right)
\end{aligned}
$$

and

$$
f\left(T_{w}, p, t\right)=\left(\begin{array}{c}
f_{1}\left(p, \bar{T}_{w, c}\right)+g_{2}\left(\bar{T}_{w, e}, t\right) u_{1} \\
f_{2}\left(\bar{T}_{w, c}, u_{2}, t\right)
\end{array}\right) .
$$

Let $p_{1}^{d}$ the desired high-pressure dynamics, and $u_{1}^{d}$ the corresponding control input. From (56), it comes that

$$
u_{1}^{d}=\frac{-A_{11} f_{1}\left(p, \bar{T}_{w, c}\right)-A_{12} f_{2}\left(\bar{T}_{w, c}, u_{2}, t\right)+{\dot{p_{1}}}^{d}}{A_{11} g_{2}\left(\bar{T}_{w, e}, t\right)}
$$

From Section 4.1 it can be easily shown that $A_{11}>0$ on the physical domain. Moreover $g_{2}($.$) does$ not vanish for $\stackrel{*}{m}_{e x h}>0$ and $T_{e x h}>\bar{T}_{w, e}$, that is when the diesel engine is on. In consequence $u_{1}^{d}$ is well defined.

Assuming from DP results analysis, that $p_{1}$ should be as high as possible (under the safety limit $\left.p^{\max }\right)$, the desired high-pressure dynamics can be stated as:

$$
\dot{p}_{1}^{d}=k_{c}\left(p_{1}^{\max }-p_{1}^{d}\right) \text { with } k_{c}>0 .
$$

Taking into account the physical constraint on by-pass opening (i.e $u_{1} \in[0,1]$ ), then it comes the following closed-loop model:

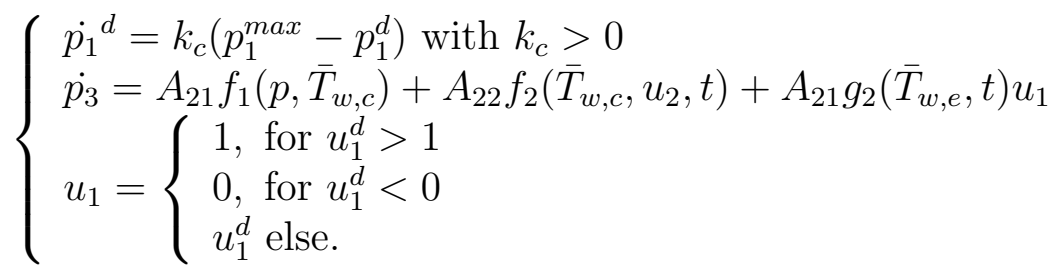

Remark 3. $k_{c}$, in the desired high-pressure dynamics (60), is a design parameter of the supervisory control. A large value of $k_{c}$ should be used to maintain $p$ as close to $p^{\text {max }}$ as possible. However, a lower value of $k_{c}$ reduces the difficulties in solving the DRTO based on the above closed-loop model.

\subsection{Dynamic real-time optimization}

The noncausal (optimal) behavior of the second control input $\stackrel{*}{m}_{A}$ shown by the DP solution detailed in Section 3.3, illustrates the potential benefit of considering the future exhaust gas conditions. As in the context of an ORC WHR system on board a diesel-electric railcar, future driving conditions can be known in advance over a short horizon, a prediction-based strategy - namely dynamic real-time optimization (DRTO) — is explored here. The formulation of the DRTO problem is similar to the moving horizon formulation used in nonlinear model-predictive control, although an economic objective is chosen to provide economically optimal operation at 
all times (see e.g Würth et al. [31]). Here, the economic objective is the net energy production along a moving horizon and the problem can be defined as follows:

$$
\begin{array}{ll} 
& \max _{u_{2}^{j}(t)} \int_{t_{0}^{j}}^{t_{f}^{j}} P_{n e t}(p, u), d t \\
\text { s.t. } & p\left(t_{0}^{j}\right)=\hat{p}^{j}
\end{array}
$$

where the (closed-loop) dynamics of $p$ is defined by system (61) and $P_{n e t}$ by equations (2)- (5). The optimization problem is solved on the time horizon $\left[t_{0}^{j}, t_{f}^{j}\right]$ (on which driving conditions are assumed to be perfectly predicted) at each sampling instant $t_{0}^{j}$ considering the (measured) value of the process output $\hat{p}^{j}$.

Notice that the state constraint (15) is directly managed by the closed-loop model (61). On the other hand, input constraints (16) are handled as described in Dufour et al. [6], that is by defining a new unconstrained decision variable $d^{j}$ using the invertible transformation:

$$
u_{2}^{j}=g\left(d^{j}\right)=g_{m o y}+g_{a m p} \tanh \left(\frac{d^{j}-g_{m o y}}{g_{a m p}}\right),
$$

where $g_{\text {moy }}=\left(u_{2}^{\max }-u_{2}^{\min }\right) / 2$ and $g_{a m p}=\left(u_{2}^{\max }+u_{2}^{\min }\right) / 2$.

The pressures trajectory, resulting from DRTO solution, provides the set-point of a low-level controller as illustrated in Figure 10. This trajectory planning is implemented on the current sampling interval, and the optimization horizon is then shifted by the sampling interval $\Delta t$. Notice that a low-level superheating controller - that is not presented here for the sake of clarity - must also be implemented. Details on the pressure and superheating low-level controllers used in this study can be found in Peralez et al. [20] and Peralez et al. [19].

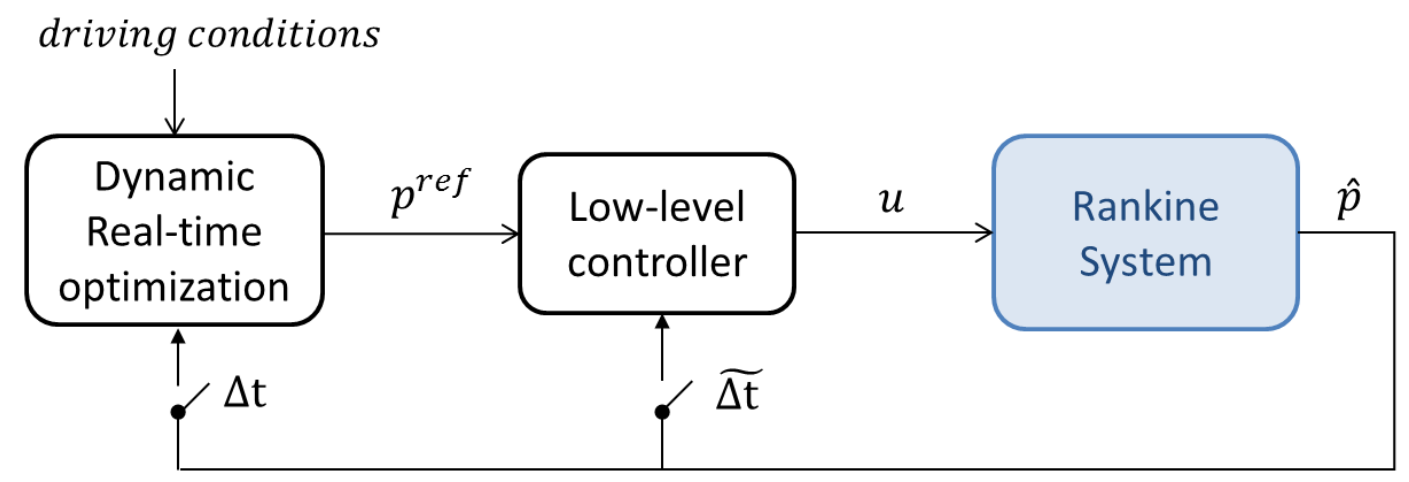

Figure 10: Two-level closed-loop control strategy. DRTO provides the ORC pressures set-point $p^{\text {ref }}$ (with a sampling time $\Delta t$ ) to the low-level controller which computes the control values - corresponding to evaporator by-pass and air fan actuators - at a (faster) sampling time $\tilde{\Delta} t$.

The relevance of the DRTO method is shown in Section 4.4. The benefits of using a dynamic model for supervisory control are evaluated by comparing DRTO with a simpler control strategy based on static optimization, akin to those presented in the studies mentioned in the beginning of the paper (Quoilin et al. [26, 25], Manente et al. [16]). In static optimization, a quasi-steady-state model derived from the dynamic model (Section 2.2), where time derivatives are set to zero, is used. 


\subsection{Application of dynamic real-time optimization}

The supervisory control structure of DRTO illustrated in Figure 10 is applied to a realistic Rankine system simulator (based on the moving-boundary principle, see e.g Peralez et al. [20]). The method described in Section 4.3 is implemented with a moving horizon length of $60 \mathrm{~s}$ and sampling time values of $\Delta t=1 \mathrm{~s}$ and $\tilde{\Delta} t=0.02 \mathrm{~s}$. Notice that the chosen value for $\Delta t$ remains small when compared to the response time of the simplified model used for the DRTO.

The benefits of using a dynamic model for the supervisory control are evaluated by comparing DRTO with the simpler control strategy based on static optimization. It is shown (Figure 11) that the increase in the amount of recovered energy $J$ is of about $7 \%$.

In order to demonstrate the relevance of the splitting approach introduced in section 4.2 , the DRTO problem with original $2 \times 2$ MIMO model formulation was also solved. It resulted in a similar level of accuracy (with an increase of about $0.1 \%$ for $J$ ), whereas the computational times were dramatically increased. Thus, for the splitting approach the average and maximum runtime per step was found to be of $0.18 s$ and $0.32 s$ whereas it was of $0.43 s$ and $0.90 s$ for the MIMO formulation. This computing performance, guarantees the real-time use of the proposed algorithm.

Notice that state variables and control inputs trajectories resulting from the - closed loop DRTO (Figure 10) are close to the - ideal - DP solution (Figure 8). Because the control model is not perfect (i.e it is different from the simulation model) unavoidable differences in the results remain.

\section{Conclusion}

In this study, an original optimal control problem is formulated in order to find the maximum power produced by an organic Rankine cycle waste heat recovery system on board a diesel-electric railcar, along a given mission profile. The development of an ad-hoc reduced-order dynamic model, validated on experimental data, makes solving the optimization problem computationally possible using a novel version of the state-of-the-art level-set DP algorithm, based on an adaptive grid.

The analysis of DP results leads to a dynamic real-time optimization problem with a single decision variable, which can be solved online and implemented as a supervisory control for the ORC system. Although providing theoretical proof of its stability is a difficult task, a future experimental implementation could demonstrate the relevance of the proposed strategy. However, the results obtained when it is applied to a realistic Rankine system simulator show a significant increase in the amount of recovered energy with respect to the benchmark provided by a simpler (but state-of-the-art) supervisory control based on static optimization.

Finally, the offline and online control strategies presented in this article are, in the author's opinion, of general interest, though they have been designed for an ORC with some distinctive - more or less favorable - features, namely in terms of cooling system, power management and mission predictability. Their application to other vehicular ORCs, on board road vehicles for instance, appears perfectly possible, though some adaptations may be needed.

\section{Acknowledgments}

The authors wish to thank ANR, the French National Research Agency, for partially funding this work within the framework of the project TRENERGY ( Train Energy Efficiency via Rankinecycle Exhaust Gas Heat Recovery", \# ANR-12-VPTT-09), and ALSTOM Transport, for providing application data. 

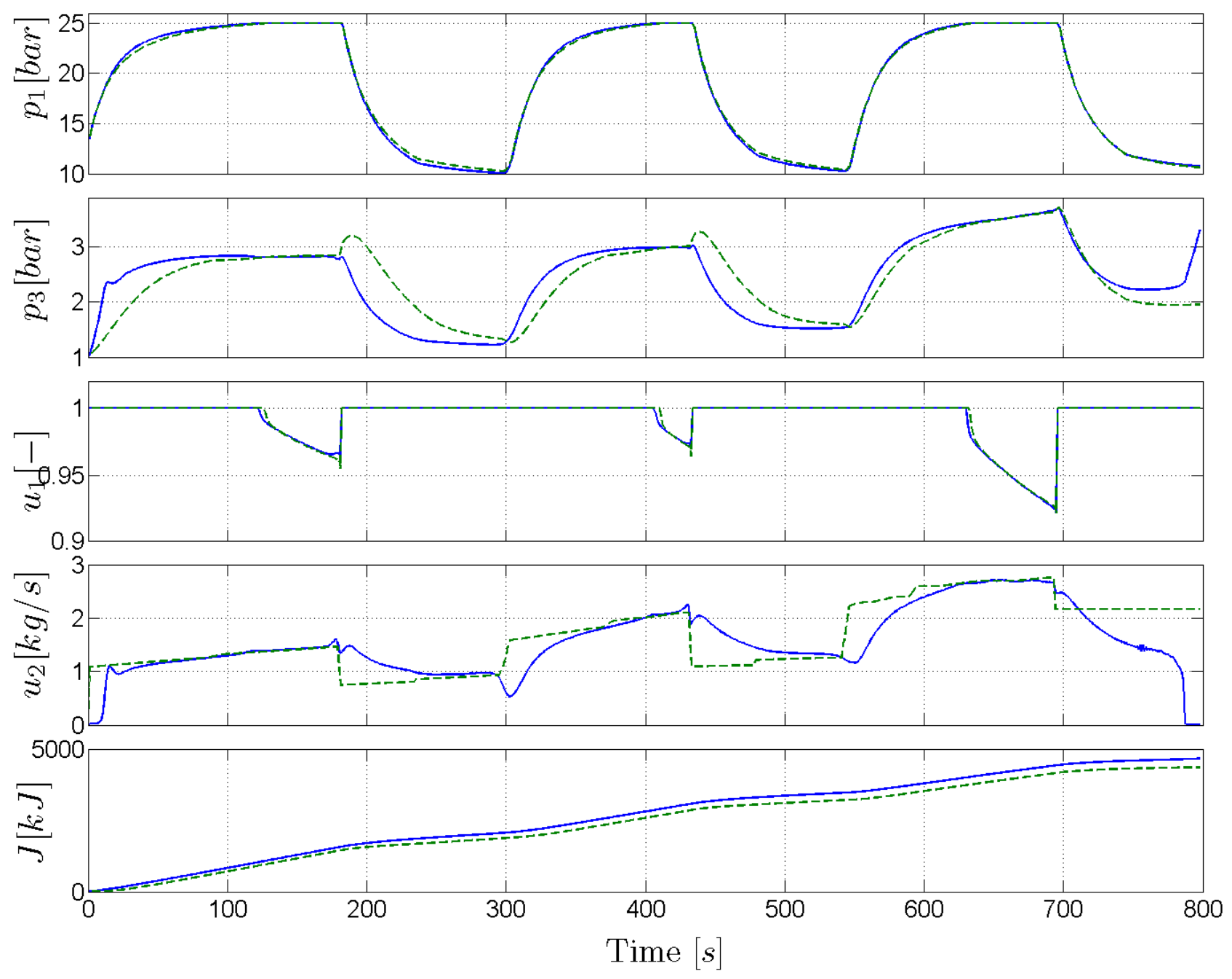

Figure 11: Result of dynamic real-time optimization (solid blue lines) compared with static optimization approach (dash green lines).

\section{References}

[1] Back, M., Terwen, S., Krebs, V., 2004. Predictive powertrain control for hybrid electric vehicles. In: 6th IFAC Symposium on Advances in Automotive Control. pp. 270 - 275.

[2] Bao, J., Zhao, L., 2013. A review of working fluid and expander selections for organic rankine cycle. Renewable and Sustainable Energy Reviews 24 (0), 325 - 342.

[3] Bellman, R., 1957. Dynamic programming. Princeton Univ. Press.

[4] Borsukiewicz-Gozdur, A., 2013. Pumping work in the organic rankine cycle. Applied Thermal Engineering 51 (1), 781-786.

[5] Cheng, T., He, X., Asada, H., 2004. Nonlinear observer design for two-phase flow heat ex- 
changers of air conditioning systems. In: American Control Conference, 2004. Proceedings of the 2004. pp. 1534-1539.

[6] Dufour, P., Touré, Y., Blanc, D., Laurent, P., 2003. On nonlinear distributed parameter model predictive control strategy: on-line calculation time reduction and application to an experimental drying process. Computers \& Chemical Engineering 27 (11), 1533 - 1542.

[7] Elbert, P., Ebbesen, S., Guzzella, L., 2013. Implementation of dynamic programming for $n$ -dimensional optimal control problems with final state constraints. IEEE Transactions on Control Systems Technology 21 (3), 924-931.

[8] Endo, T., Kawajiri, S., Kojima, Y., Takahashi, K., Baba, T., Ibaraki, S., Takahashi, T., Shinohara, M., 2007. Study on maximizing exergy in automotive engines. SAE Technical Paper 2007-01-0257.

[9] Feru, E., Kupper, F., Rojer, C., Seykens, X., Scappin, F., Willems, F., Smits, J., Jager, B. D., Steinbuch, M., 2013. Experimental validation of a dynamic waste heat recovery system model for control purposes. SAE Technical Paper 2013-01-1647.

[10] Guzzella, L., Sciarretta, A., 2007. Vehicle propulsion systems. Springer.

[11] Hammouri, H., Nadri, M., 2013. An observer design for a class of implicit systems. Systems \& Control Letters 62 (3), $256-261$.

[12] Horst, T. A., Rottengruber, H. S., Seifert, M., Ringler, J., 2013. Dynamic heat exchanger model for performance prediction and control system design of automotive waste heat recovery systems. Applied Energy 105 (0), 293 - 303.

[13] Horst, T. A., Tegethoff, W., Eilts, P., Koehler, J., 2014. Prediction of dynamic rankine cycle waste heat recovery performance and fuel saving potential in passenger car applications considering interactions with vehicles' energy management. Energy Conversion and Management $78(0), 438-451$.

[14] Hou, G., Sun, R., Hu, G., Zhang, J., 2011. Supervisory predictive control of evaporator in organic rankine cycle (ORC) system for waste heat recovery. In: Advanced Mechatronic Systems (ICAMechS). pp. 306-311.

[15] Lee, J. H., Wong, W., 2010. Approximate dynamic programming approach for process control. Journal of Process Control 20 (9), 1038-1048.

[16] Manente, G., Toffolo, A., Lazzaretto, A., Paci, M., 2013. An organic rankine cycle off-design model for the search of the optimal control strategy. Energy 58 (1), 97 - 106.

[17] McKinley, T. L., Alleyne, A. G., 2008. An advanced nonlinear switched heat exchanger model for vapor compression cycles using the moving-boundary method. International Journal of Refrigeration 31 (7), 1253 - 1264.

[18] Merz, F., Sciarretta, A., Dabadie, J.-C., Serrao, L., 2012. On the optimal thermal management of hybrid-electric vehicles with heat recovery systems. Oil \& Gas Science and Technology-Revue d'IFP Energies nouvelles 67 (4), 601 - 612. 
[19] Peralez, J., Nadri, M., Dufour, P., Tona, P., Sciarretta, A., 2014. Control design for an automotive turbine rankine cycle system based on nonlinear state estimation. In: 2014 IEEE Conference on Decision and Control (CDC). pp. 3316-3321.

[20] Peralez, J., Tona, P., Lepreux, O., Sciarretta, A., Voise, L., Dufour, P., Nadri, M., 2013. Improving the control performance of an organic rankine cycle system for waste heat recovery from a heavy-duty diesel engine using a model-based approach. In: 2013 IEEE Conference on Decision and Control (CDC). pp. 6830-6836.

[21] Peralez, J., Tona, P., Sciarretta, A., Dufour, P., Nadri, M., 2012. Towards model-based control of a steam rankine process for engine waste heat recovery. In: IEEE Vehicle Power and Propulsion Conference (VPPC). pp. 289-294.

[22] Peralez, J., Tona, P., Sciarretta, A., Dufour, P., Nadri, M., 2014. Optimal control of a vehicular organic rankine cycle via dynamic programming with adaptive discretization grid. In: 19th IFAC World Congress. pp. 5671-5678.

[23] Petit, N., Sciarretta, A., 2011. Optimal drive of electric vehicles using an inversion-based trajectory generation approach. In: 18th IFAC World Congress. pp. 14519-14526.

[24] Powell, W. B., 2007. Approximate Dynamic Programming: Solving the curses of dimensionality. Vol. 703. John Wiley \& Sons.

[25] Quoilin, S., Aumann, R., Grill, A., Schuster, A., Lemort, V., Spliethoff, H., 2011. Dynamic modeling and optimal control strategy of waste heat recovery Organic Rankine Cycles. Applied Energy 88 (6), 2183-2190.

[26] Quoilin, S., Declaye, S., Tchanche, B. F., Lemort, V., 2011. Thermo-economic optimization of waste heat recovery organic rankine cycles. Applied Thermal Engineering 31 (14-15), 28852893.

[27] Sprouse, C., Depcik, C., 2013. Review of organic rankine cycles for internal combustion engine exhaust waste heat recovery. Applied thermal engineering 51 (1-2), 711-722.

[28] Stanton, D., 2013. Systematic development of highly efficient and clean engines to meet future commercial vehicle greenhouse gas regulations. SAE Int. J. Engines 6 (3), 1395-1480.

[29] Sundström, O., Ambühl, D., Guzzella, L., 2010. On implementation of dynamic programming for optimal control problems with final state constraints. Oil \& Gas Science and TechnologyRevue de l'Institut Français du Pétrole 65 (1), 91-102.

[30] Willems, Frank, Kupper, Frank, Rascanu, George, Feru, Emanuel, 2014. Integrated energy and emission management for diesel engines with waste heat recovery using dynamic models. Oil Gas Sci. Technol. - Rev. IFP Energies nouvelles. doi:10.2516/ogst.2013.210.

[31] Würth, L., Hannemann, R., Marquardt, W., 2009. Neighboring-extremal updates for nonlinear model-predictive control and dynamic real-time optimization. Journal of Process Control 19 (8), $1277-1288$. 
[32] Xie, H., Yang, C., 2013. Dynamic behavior of rankine cycle system for waste heat recovery of heavy duty diesel engines under driving cycle. Applied Energy 112 (0), 130 - 141.

[33] Zhang, J., Zhou, Y., Gao, S., Hou, G., 2012. Constrained predictive control based on state space model of organic rankine cycle system for waste heat recovery. In: Chinese Control and Decision Conference (CCDC). pp. $230-234$. 


\section{Appendix A. A simple energy management example}

To illustrate the benefits of the new algorithm, a first optimal control problem with an analytic solution is studied. It concerns energy management for an electric vehicle.

\section{Appendix A.1. System description}

Neglecting the aerodynamic friction and considering the road slope null, the simplified continuoustime dynamics of the vehicle in the longitudinal direction $x$ can be written as in Petit and Sciarretta [23]:

$$
\dot{x}=v, \dot{v}=h_{1} u-h_{0},
$$

where the control variable $u$ is a percent torque demand, $h_{0}$ and $h_{1}$ are constant parameters depending on the rolling resistance coefficient, the motor maximum torque, vehicle mass, transmission ratio and wheel radius.

The on-board electric power consumption can be written as

$$
P_{m}=b_{1} u v+b_{2} u^{2}
$$

\section{Appendix A.2. Optimal control problem}

The aim is to find a control strategy that minimizes the power consumption under the constraints that the vehicle must reach a destination point at a distance $D$ in a given time $t_{f}$ starting from a given point, at rest. In order to formulate this problem for DP solving, the continuoustime model is discretized using an Euler forward approximation with a time step $T_{s}$. The optimal control problem can be formulated as follows:

$$
\begin{array}{r}
\min _{u_{k} \in[-2,2]} \sum_{k=0}^{N-1}\left(b_{1} u_{k} v_{k}+b_{2} u_{k}^{2}\right) T_{s} \\
v_{k+1}=v_{k}+\left(h_{1} u_{k}-h_{0}\right) T_{s} \\
x_{k+1}=x_{k}+v_{k} T_{s} \\
x_{0}=0, v_{0}=0 \\
0 \leq x_{k} \leq \bar{x}, 0 \leq v_{k} \leq \bar{v} \\
D \leq x_{N} \leq \bar{x}, 0 \leq v_{N} \leq \epsilon_{v},
\end{array}
$$

where $N=\frac{t_{f}}{T_{s}}$. The final constraints are formulated in equations (A.8). In the following, a particular solution is sought for a final distance $D=200$ and a final time $t_{f}=60$. The time discretization is chosen to be $T_{s}=0.2 s$ (then $N=300$ ), with $\bar{x}=205, \bar{v}=8, \epsilon_{v}=0.3$.

\section{Appendix A.3. DP solution}

The problem stated above is solved using the basic, the original level-set and the level-set with two-step discretization DP algorithms.

State variable spaces are first discretized respectively with $N_{v}=81$ and $N_{x}=106$, while the control space is discretized with $N_{u}=81$. Figure A.12 shows the resulting system trajectory and the corresponding control inputs for each of the three algorithms. They are compared to the analytic solution (a parabola) presented by Petit and Sciarretta [23]. The results obtained using the new algorithm are closer to the analytic solution, even with a relatively coarse discretization. 


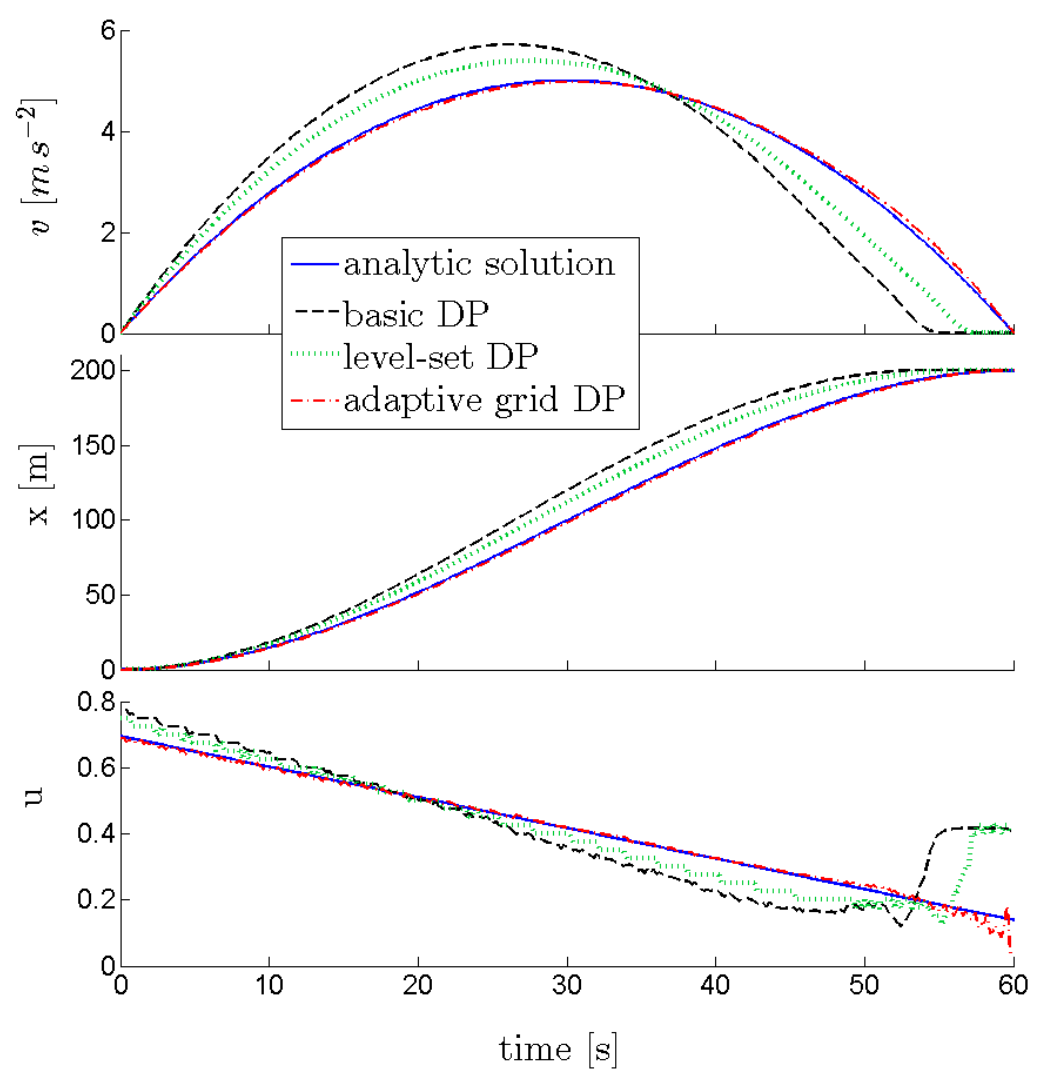

Figure A.12: Vehicle trajectory and control inputs when solving the problem with $N_{v}=81, N_{x}=106$ and $N_{u}=81$

In term of cost, the relative mean errors of the three algorithms are found to be of $1.4 \%, 0.78 \%$ and $0.19 \%$ respectively.

The evolution of backward-reachable space boundaries, where level-set function vanishes, helps to analyze those results. Figure A.13 shows that in the first backward steps, the optimal trajectory $\left(x^{o}, v^{o}\right)$ is close to $\tilde{I}_{k}$ estimations. Here, for accurate estimation of boundaries it is important not to consider reachable points close to the optimal solution as infeasible. After those first backward steps, the optimal trajectory moves away from the boundaries. However, the reachable state space remain restricted. Thus, the second discretization step of the proposed algorithm can improve the accuracy of cost-to-go function.

Two-step discretization involves a larger computational effort for a given set of parameters $\left(N_{v}, N_{x}, N_{u}\right)$. This computation cost can be expressed in terms of number of model calls for evaluation. For example, for the level of discretization used above, basic and original level-set DP both require about $2.085 \cdot 10^{8}$ model calls whereas the new algorithm requires about $2.85 \cdot 10^{8}$. For a fair comparison, algorithm accuracies are compared for different levels of discretization. Results are shown in Figure A.14. The adaptive-grid DP algorithm attains acceptable accuracies for a much lower number of calls. 


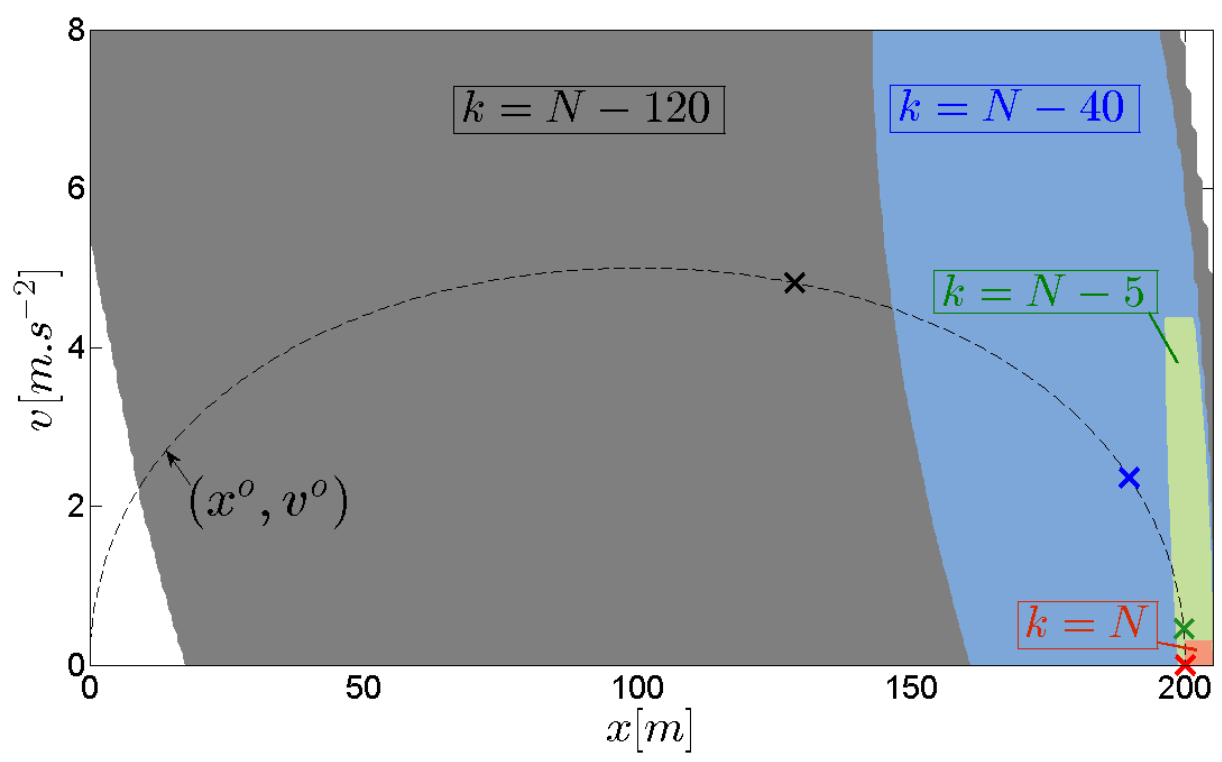

Figure A.13: Optimal trajectory $\left(x^{o}, v^{o}\right)$ and evolution of backward-reachable space (evaluated by adaptive-grid $\mathrm{DP})$.

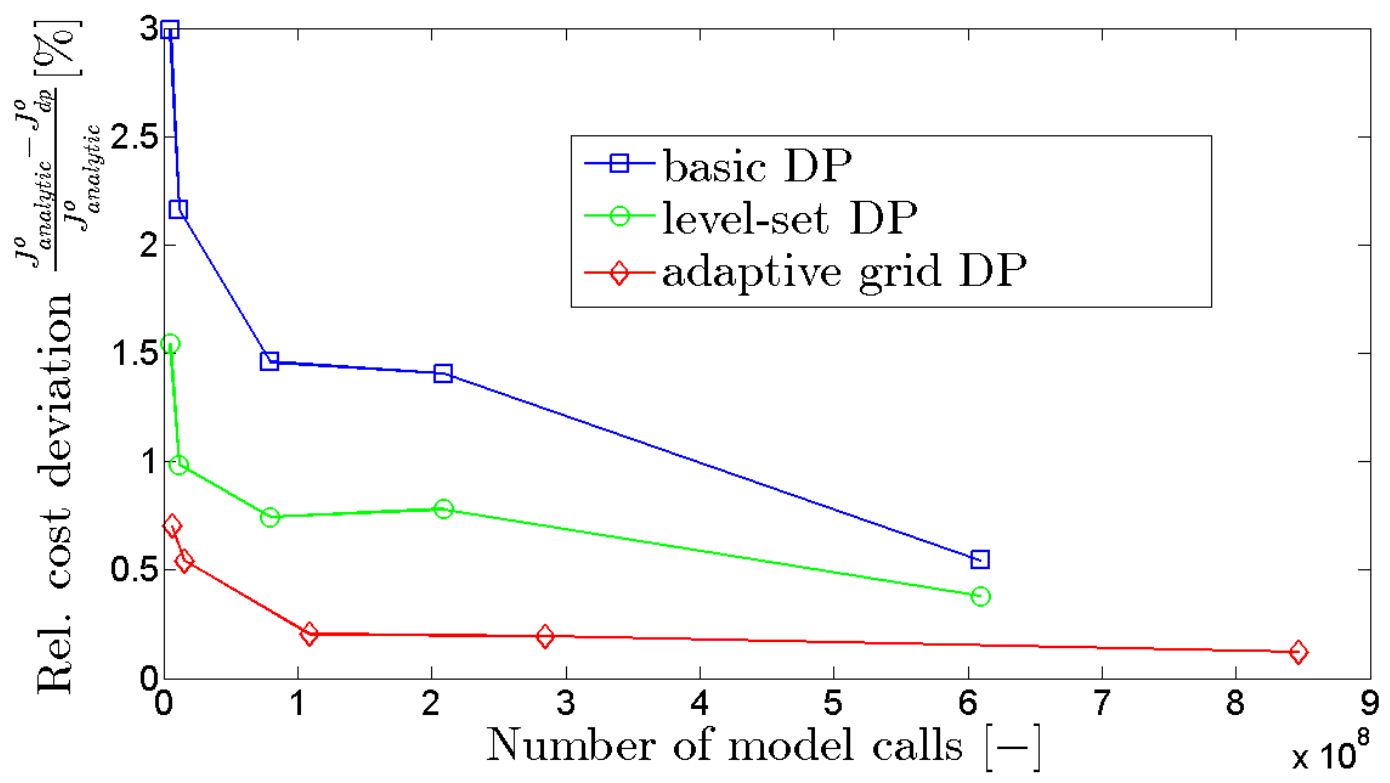

Figure A.14: Algorithm accuracies vs number of model calls for the simple energy management example. 